\title{
Insights into in vivo adipocyte differentiation through cell-specific labeling in zebrafish
}

\author{
Paola Lepanto ${ }^{1, \pm, \S}$, Florencia Levin-Ferreyra ${ }^{1, *, \pm}$, Uriel Koziol ${ }^{2}$, Leonel Malacrida ${ }^{3,4}$ and José L. Badano ${ }^{1, \pm}$
}

\begin{abstract}
White adipose tissue hyperplasia has been shown to be crucial for handling excess energy in healthy ways. Though adipogenesis mechanisms have been underscored in vitro, we lack information on how tissue and systemic factors influence the differentiation of new adipocytes. While this could be studied in zebrafish, adipocyte identification currently relies on neutral lipid labeling, thus precluding access to cells in early stages of differentiation. Here we report the generation and analysis of a zebrafish line with the transgene fabp4a(2.7):EGFPcaax. In vivo confocal microscopy of the pancreatic and abdominal visceral depots of transgenic larvae, revealed the presence of labeled mature adipocytes as well as immature cells in earlier stages of differentiation. Through co-labeling for blood vessels, we observed a close interaction of differentiating adipocytes with endothelial cells through cell protrusions. Finally, we implemented hyperspectral imaging and spectral phasor analysis in Nile Red-labeled transgenic larvae and revealed the lipid metabolic transition towards neutral lipid accumulation of differentiating adipocytes. Altogether our work presents the characterization of a novel adipocyte-specific label in zebrafish and uncovers previously unknown aspects of in vivo adipogenesis.
\end{abstract}

This article has an associated First Person interview with the first author of the paper.

\section{KEY WORDS: Zebrafish, Adipocyte, Nile Red, Blood vessels}

\section{INTRODUCTION}

White adipose tissue (WAT) is present in mammals as well as in the other vertebrates, in the form of anatomically and functionally distinct depots (Zwick et al., 2018). It is formed by adipocytes, adipocyte precursors and macrophages surrounded by a collagenrich extracellular matrix, and is highly vascularized and innervated. In humans, both visceral and subcutaneous central WAT depots

\footnotetext{
${ }^{1}$ Human Molecular Genetics Lab, Institut Pasteur de Montevideo, Montevideo, Mataojo 2020, CP11400, Uruguay. ²Sección Biología Celular, Facultad de Ciencias, Universidad de la República, Montevideo, Igua 4225, CP11400, Uruguay. ${ }^{3}$ Advanced Bioimaging Unit, Institut Pasteur de Montevideo and Universidad de la República, Montevideo, Mataojo 2020, CP11400, Uruguay. ${ }^{4}$ Departamento de Fisiopatología, Hospital de Clínicas, Facultad de Medicina, Universidad de la República, Montevideo, Av. Italia s/n, CP11600, Uruguay. *Present address: Center for Stem Cell \& Regeneration, Baylor College of Medicine, One Baylor Plaza, Houston, TX 77030, USA.

tshared first authorship

§authors for correspondence (jbadano@pasteur.edu.uy; plepanto@pasteur.edu.uy)
}

(iD P.L., 0000-0003-3027-4398; F.L.-F., 0000-0003-0395-5331; U.K., 0000-00029699-4964; L.M., 0000-0001-6253-9229; J.L.B., 0000-0002-0706-8652

This is an Open Access article distributed under the terms of the Creative Commons Attribution License (https://creativecommons.org/licenses/by/4.0), which permits unrestricted use, distribution and reproduction in any medium provided that the original work is properly attributed.

Received 1 April 2021; Accepted 10 August 2021 primarily play energy storage and endocrine functions, which are of central importance in the regulation of energy homeostasis. Thus, WAT dysfunction, which is usually associated with obesity, contributes to the development of metabolic syndrome associated-diseases such as type II diabetes, dyslipidemia and nonalcoholic fatty liver disease (Longo et al., 2019). Meanwhile, other depots such as those in the dermis, bone marrow and mammary gland, contribute to regulation of local innate immunity and to the repair of adjacent tissues (Zwick et al., 2018).

In humans, the localization and mode of remodeling of adipose tissue have been associated with healthy or pathological phenotypes (Hepler and Gupta, 2017). Macroscopically, the expansion of subcutaneous WAT (SAT) is considered healthier than the growth of visceral WAT (VAT), as well as its localization in peripheral (extremities) versus central (abdomen in men; abdomen and hips in women) depots. Also, the accumulation of fat can occur in previously existing mature adipocytes (hypertrophy) or in newly differentiated cells (hyperplasia). Importantly, different lines of evidence support an association between adipose tissue hyperplasia with a healthier state as compared to hypertrophy (Vishvanath and Gupta, 2019). Adipogenesis is the process whereby stem cell-like precursors become committed into pre-adipocytes, which then differentiate into mature adipocytes. While initial formation of adipogenic progenitors occur in hematopoietic tissues (Hudak et al., 2014), adult progenitors reside locally associated to blood vessels of adipose tissue (Hilgendorf et al., 2019; Tang et al., 2008). Tissue environment and cellular composition may influence the differentiation of these locally residing progenitors (for example see Schwalie et al., 2018). Thus, the study of adipogenesis and its relationship with other elements in the tissue in vivo, is critical to understand normal and pathological processes.

Work on cultured cells has provided key information about transcriptional regulation of adipogenesis (Bahmad et al., 2020). Meanwhile studies in mice have been conducted to analyze the developmental origin of adipocyte progenitors (Hepler and Gupta, 2017). More recently, the use of zebrafish to analyze adipose tissue biology has captured attention as it promises to enable the study of the tissue and its cellular biology in vivo. Zebrafish develops only white adipose tissue, which first appears in visceral depots in early larval stages (Flynn et al., 2009; Minchin and Rawls, 2017b). In contrast, mice develop first SAT depots in embryonic stages while VAT appears postnatally (Hudak et al., 2014). Importantly, however, zebrafish adipocytes show the same subcellular characteristics, gene expression patterns and final distribution (visceral and subcutaneous) as in mammals (Flynn et al., 2009). Moreover, it has been reported that factors affecting body fat distributions in humans have comparable effects in zebrafish (Loh et al., 2020; Minchin et al., 2015). Thus, taking advantage of its fast external development and optical transparency, zebrafish is an ideal system to study cellular and tissular aspects of WAT development. 
Current methods to label adipose tissue in vivo rely on the use of lipophilic dyes such as LipidTOX or Nile Red (Minchin and Rawls, 2017a). Nile Red is particularly useful because its absorption and emission spectral characteristics are modified according to the polarity of the environment surrounding the probe (Greenspan and Fowler, 1985). The emission of Nile Red in the context of neutral lipids is blue-shifted in comparison to when it is in the presence of polar lipids. This spectroscopic characteristic has been extensively used to label lipid droplets and to estimate the amount of adipose tissue in live larvae as well as to classify depots (Minchin and Rawls, 2017b). However, Nile Red stains all cell membranes, including the endoplasmic reticulum in which biogenesis of lipid droplets takes place (Olzmann and Carvalho, 2019). Lipid stores are composed of neutral lipids such as triacylglycerols and sterol esters, while polar lipids are present during droplet formation as well as during lipolysis. Maulucci et al. developed an approach to generate a lipid metabolic index using hyperspectral imaging of Nile Red and spectral phasor analysis (Di Giacinto et al., 2018; Maulucci et al., 2018). Thus, this method allowed them to differentiate among cells forced to carry out lipid movement (lipid storage or lipolysis) and those in a resting state. The application of this method to live larvae as well as the study of the interaction of adipocytes with other cells in the tissue would require to specifically label adipocytes independently of their fat load.

To address this issue we decided to generate an adipocyte specific reporter zebrafish line. Up to date, no factor has been identified that is expressed in all fat depots in mice (Cleal et al., 2017). However, there are several genes that are upregulated in adipocytes in different stages during differentiation, like those used extensively in cell culture of mammalian cells to monitor differentiation progress (Tang and Lane, 2012). As expected, much less information is available from zebrafish. We therefore selected early and late genes that are commonly used as adipocyte differentiation markers in mammalian cell culture models and with previous evidence of being expressed in zebrafish adipose tissue (Imrie and Sadler, 2010), cloned their putative promoter regions and generated transgenesis constructs. We show here that a fabp $4 a$ (previously called fabp 11a) $-2.7 \mathrm{~kb}$ proximal genomic region from zebrafish effectively drives the expression of EGFP in adipocytes both previous to and during the accumulation of fat. Membrane tagging of EGFP allowed us to observe the early interaction of adipocytes with blood vessels through adipocyte membrane protrusions. Furthermore, we adapted the method of Maulucci et al. by incorporating a three-component analysis in the phasor plot, which enabled us to analyze the lipid metabolism of EGFP-positive cells in live larvae before the formation of lipid droplets. Thus, this new zebrafish transgenic line is a valuable tool which will open new possibilities to study adipocytes and adipose tissue biology in vivo.

\section{RESULTS}

\section{fabp4a(-2.7):EGFPcaax transgene is expressed in early and mature adipocytes}

Based on previously reported data on cell culture models and expression patterns in zebrafish we selected four different genes to work with: adipoqb, cebpo, $c f d, f a b p 4 a$. All of them were previously reported to be expressed in zebrafish adipose tissue in larvae and/or adults (Imrie and Sadler, 2010). Taking into account our analysis of the promoter regions, we cloned approximately $2 \mathrm{~kb}$ of the proximal part of the zebrafish promoter for each gene (see Materials and Methods), and generated transgenesis constructs using the Tol2 system bearing the cardiac light chain myosin reporter gene, cmlc2:GFP, as an early selection marker (Fig. 1A). These constructs were injected together with mRNA coding for
Tol2 transposase in the cytoplasm of one-cell stage embryos. Twenty-four hours post-fertilization (hpf) embryos with GFP expressing-cells in their hearts were selected for further breeding. We then analyzed larvae of 15-21 days post-fertilization (dpf) in the stereomicroscope, and observed the presence of labeled cells for the constructions with cebp $\alpha$ and fabp $4 a$ promoters. However, only in the latter case the EGFPcaax signal coincided with lipid droplets in mature adipocytes recognizable through transmitted light. Moreover, only in the case of larvae injected with the fabp $4 a$ (-2.7):EGFPcaax construct we observed mature adipocytes labeled along several generations (Fig. 1B). Therefore, we decided to continue working only with the fabp $4 a(-2.7)$ :EGFPcaax line.

First, to assess EGFP expression in live fabp4a(-2.7):EGFPcaax larvae we stained individuals of different stages with the lipophilic dye LipidTOX-Red and analyzed them using epifluorescence microscopy. We observed EGFPcaax signal in the surface of mature adipocytes, both in the pancreatic and abdominal depots (PVAT and AVAT, respectively) (Fig. 1B, asterisks). Of note, expression levels varied among cells and this effect remained even after several outcrosses with the wild-type fish line. We also analyzed other depots present in $27 \mathrm{dpf}$ larvae (renal VAT, ocular SAT, lateral SAT and anal fin ray SAT) and detected cells with expression of EGFPcaax (Fig. S1). Interestingly, besides mature adipocytes with readily visible lipid droplets, we observed EGFPcaax-positive (EGFP+) cells that had smaller lipid accumulations as well as cells that had no detectable LipidTOX-Red signal (Fig. 1B, single and double arrows, respectively). Based on the fact that mammalian fabp 4 is expressed during adipocyte differentiation (Tang and Lane, 2012), these results indicated that EGFP+ cells with no visible lipid droplets (to which we refer as EGFP+/LD- from now on) were likely adipocytes at initial stages of differentiation.

\section{fabp4a(-2.7):EGFPcaax transgene expression pattern recapitulates the adipose tissue expression domain of endogenous fabp4a}

Embryonic fabp $4 a$ expression has been reported to be restricted to the lens, midbrain and the blood vessels of the head and trunk in 48 hpf embryos (Liu et al., 2007). Meanwhile, 15 dpf larvae present fabp $4 a$ expression in trunk vessels and in early adipocytes (Flynn et al., 2009). To determine the expression pattern of the fabp $4 a$ (-2.7):EGFPcaax transgene, we analyzed several developmental stages and compared it with endogenous fabp $4 a$ expression. For this, endogenous fabp $4 a$ expression was assessed using fluorescent wholemount in situ hybridization (WMISH), and fabp 4a(-2.7): EGFPcaax transgene expression through immunolabeling with an anti-GFP antibody.

First, we checked the specificities of WMISH probes using flil: EGFP transgenic embryos, which express EGFP in blood vessels, useful as an anatomical reference. For that, $2 \mathrm{dpf}$ wild-type embryos were fixed and processed for WMISH as described in the Materials and Methods section. As specificity controls, we used the fabp $4 a$ sense probe (negative control) and an antisense probe for slit2 (additional specificity control). To detect EGFP, we performed a final immunolabeling step. We observed a clear signal corresponding to fabp $4 a$ transcripts when the WMISH was performed with the antisense probe, which co-localized with fli1:EGFP immunodetection almost completely (Fig. S2; also note the presence of brain cells positive for fabp $4 a$ antisense probe without fli1:EGFP labeling). Neither of the other two probes generated similar patterns: no specific signal was observed with the $f a b p 4 a$ sense probe while the slit 2 antisense probe labeled the ventro-medial part of the neural tube as reported previously (Davison and Zolessi, 2021). These results corroborated the specificity 
A

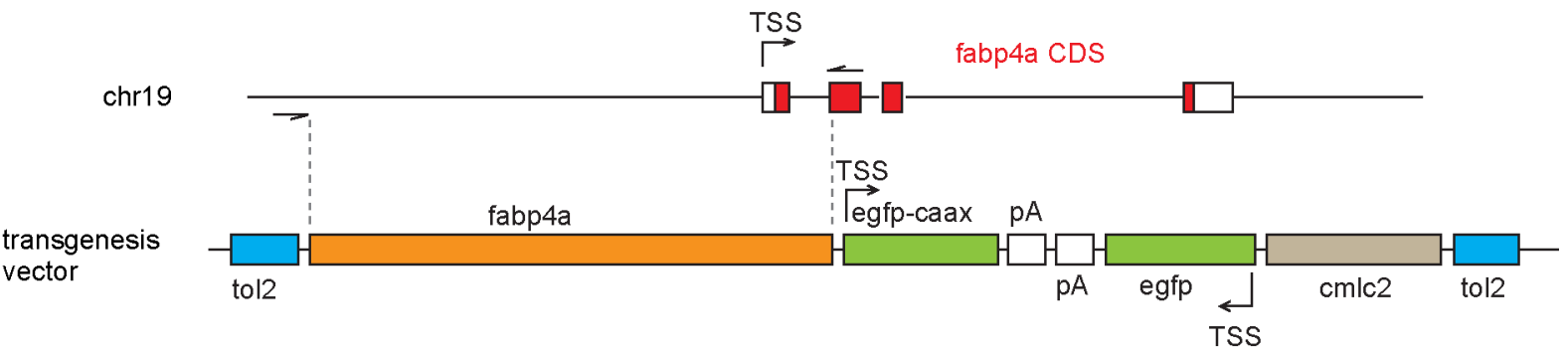

B
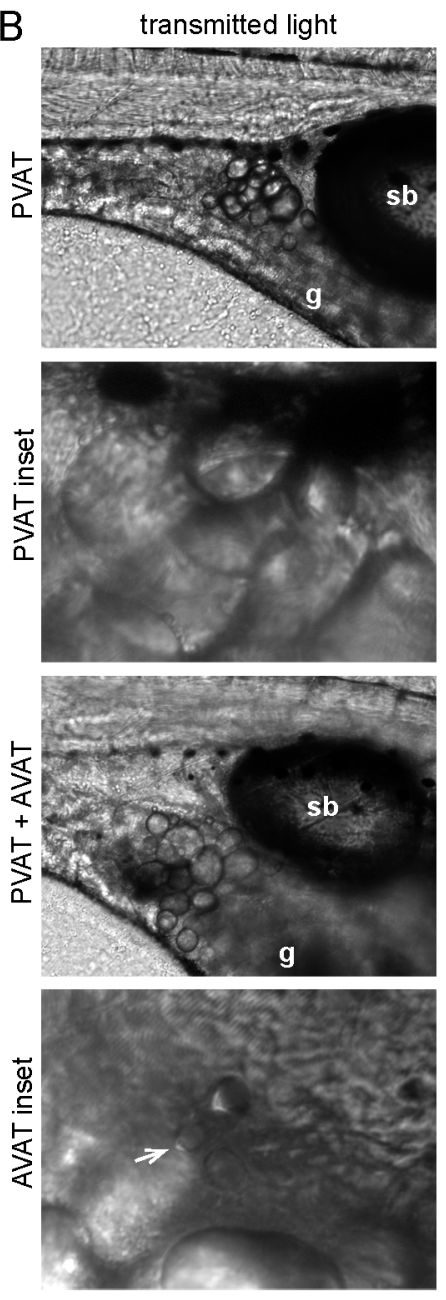
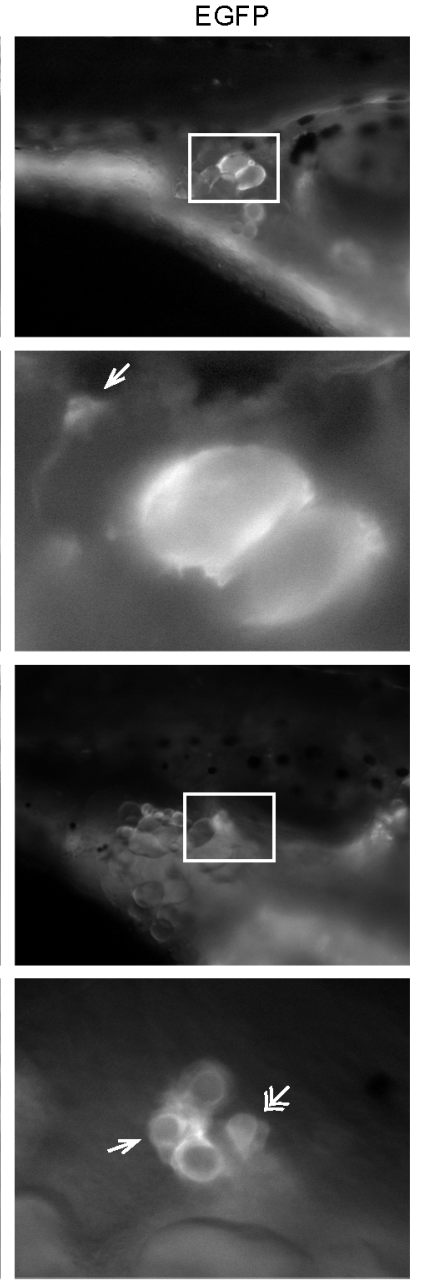
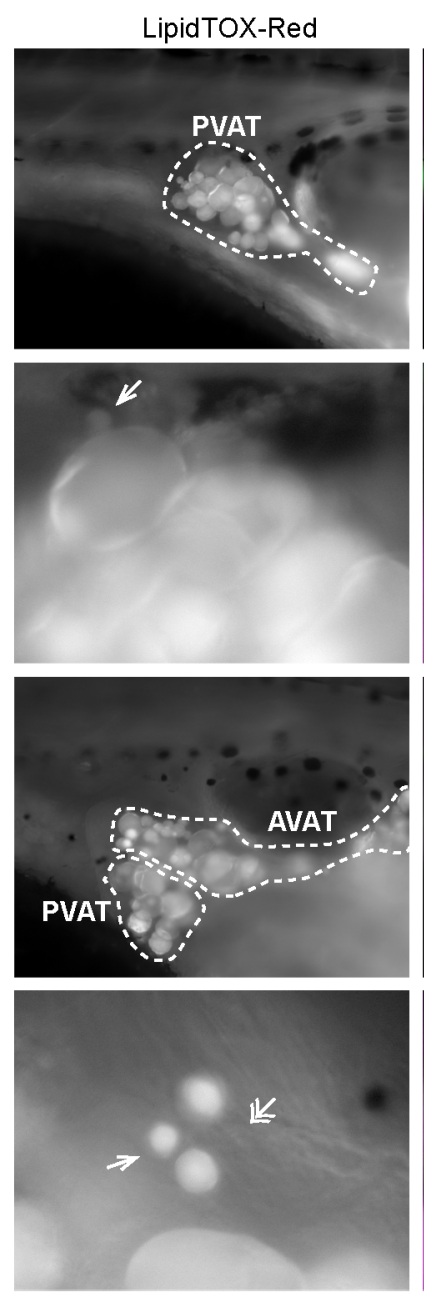

EGFP / LipidTOX-Red
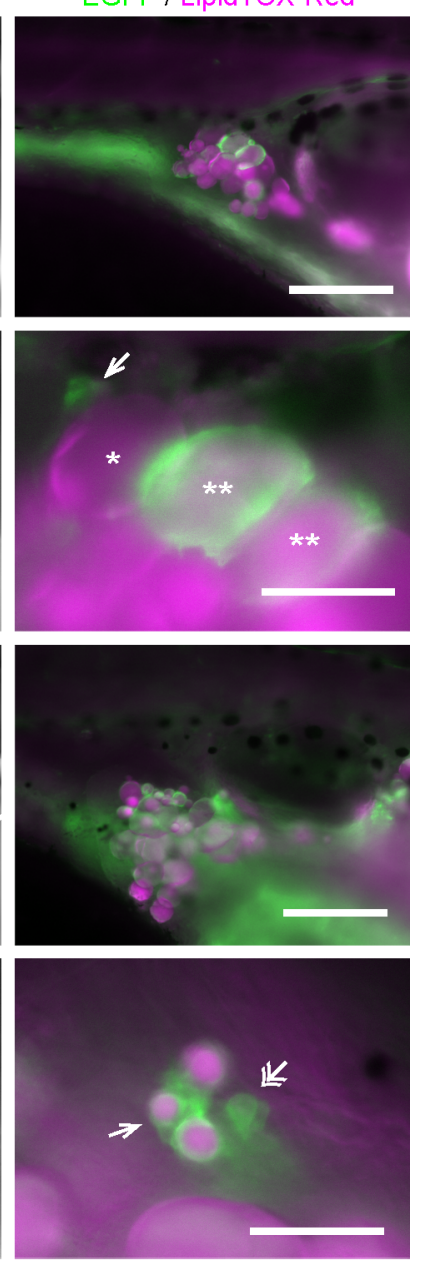

Fig. 1. fabp4a(-2.7):EGFPcaax is expressed in early and mature adipocytes. (A) The upper scheme shows the endogenous fabp4a gene in chromosome 19 with the transcription start site (TSS), exons (boxes), introns, and the coding sequence (CDS) in red. The cloned region is denoted between dashed lines. The lower scheme represents the vector used for transgenesis (tol2: tol2 sites; pA: SV40_late_polyA; cmlc2: cardiac myosin light chain 2 upstream region). (B) Epifluorescence microscopy images of live fabp4a(-2.7):EGFPcaax larvae from the incross of the F3 generation labeled with LipidTOX-Red. EGFP+ cells were present in the PVAT and AVAT depots. Asterisks indicate mature adipocytes stained with LipidTOX-Red with low $\left({ }^{*}\right)$ or high EGFPcaax expression $\left({ }^{* *}\right)$. Single arrows denote early adipocytes expressing EGFPcaax with small lipid droplets. Double arrows indicate early adipocytes with EGFPcaax expression without LipidTOX-Red staining. sb, swim bladder; g, gut. Scale bars: B: panoramic views: $200 \mu \mathrm{m}$; insets: $50 \mu \mathrm{m}$.

of the fabp $4 a$ antisense probe and validated the post-WMISH immunofluorescence procedure and reagents.

To compare the distribution of transgenic fabp $4 a(-2.7)$ : EGFPcaax expression with that of endogenous fabp $4 a$ in larvae, we performed WMISH in individuals of $21 \mathrm{dpf}$, immunolabeled them with anti-GFP and analyzed them in toto using confocal microscopy. Endogenous expression was observed in the PVAT and AVAT areas with the fabp $4 a$ antisense probe, localizing within cells with EGFPcaax expression (Fig. 2A). While signal was observed with the fabp $4 a$ sense probe, likely due to the thickness of the sample hindering an efficient clearance of non-specific labeling during washing steps, it showed less fluorescence intensity than the anti-sense probe and a different subcellular localization (Fig. 2B,C). Also, EGFPcaax expression was evidenced in pigment cells in live embryos (Fig. S3). This expression pattern was not described before for endogenous $f a b p 4 a$ and, in larvae processed for WMISH, expression of endogenous fabp $4 a$ was not observed in superficial pigment cells (Fig. 2D, single arrows). Instead, we did observe staining for endogenous fabp $4 a$ in blood vessels (Fig. 2D, double arrows). In conclusion, the expression pattern of the transgene recapitulates the endogenous pattern of fabp $4 a$ in the adipose tissue, but not within blood vessels or in the brain. 
A
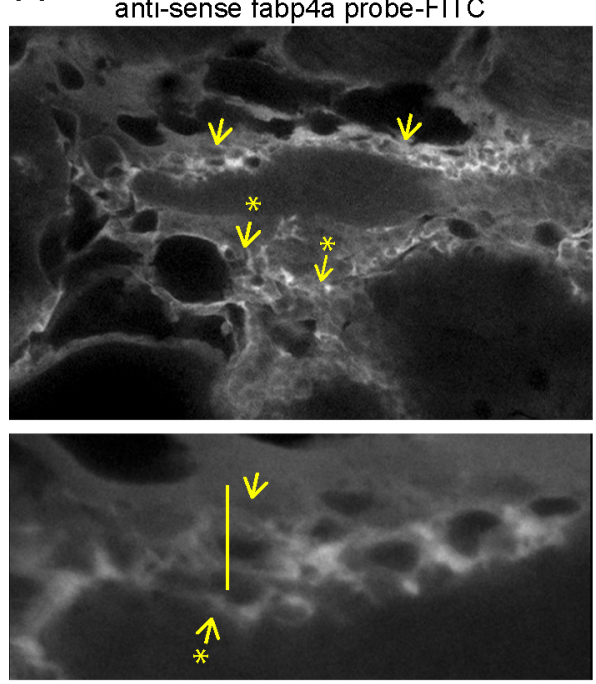

B
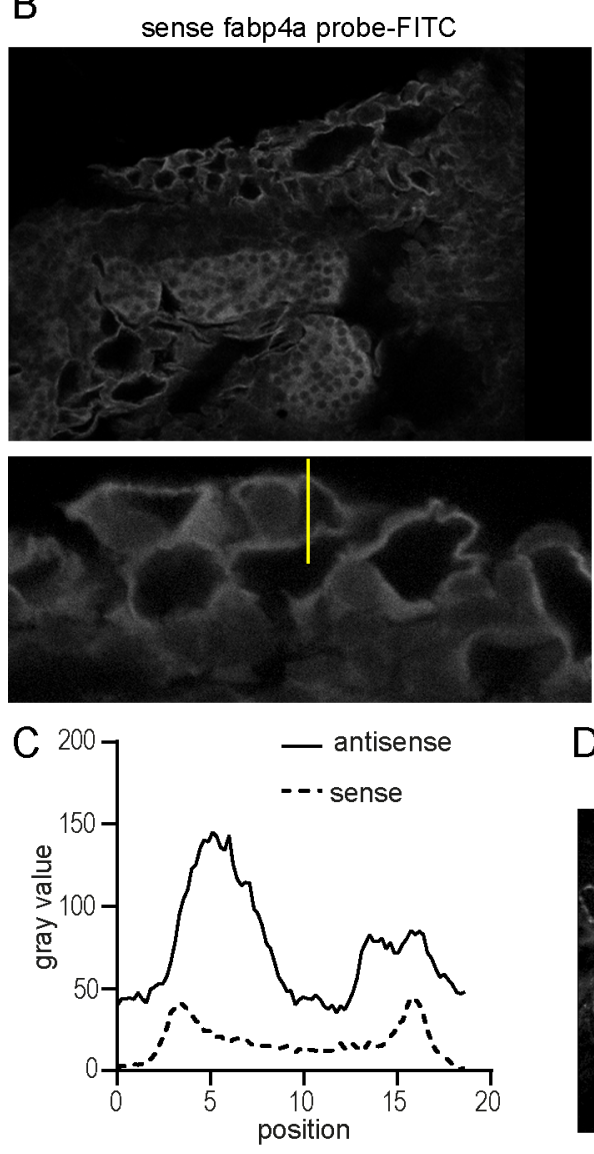

fabp4a:EGFPcaax - aGFP-TMR
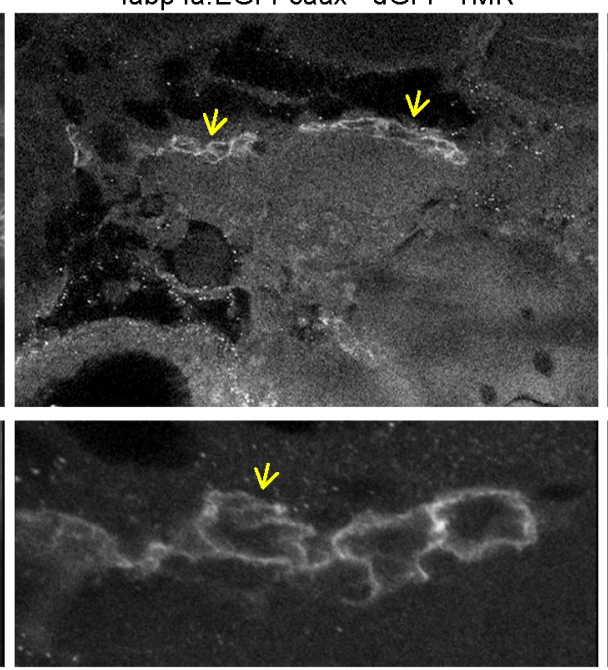

fabp4a:EGFPcaax - aGFP-TMR
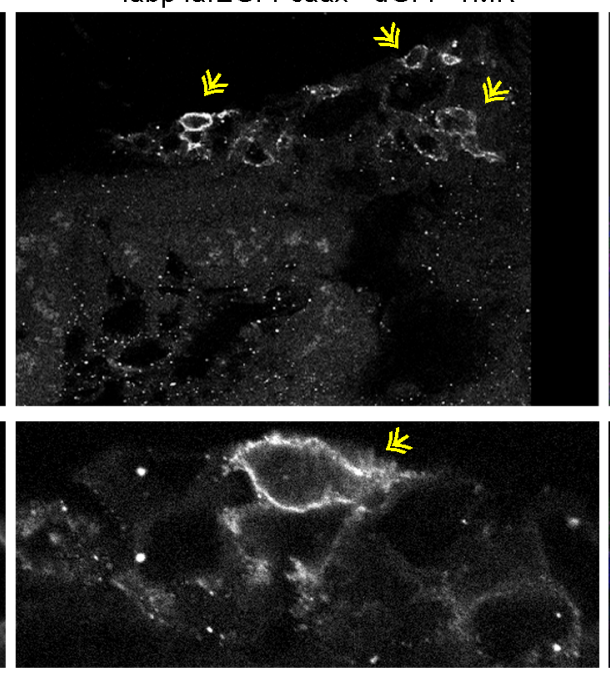

anti-sense fabp4a probe-FITC / fabp4a:EGFPcaax - aGFP-TMR / nuclei
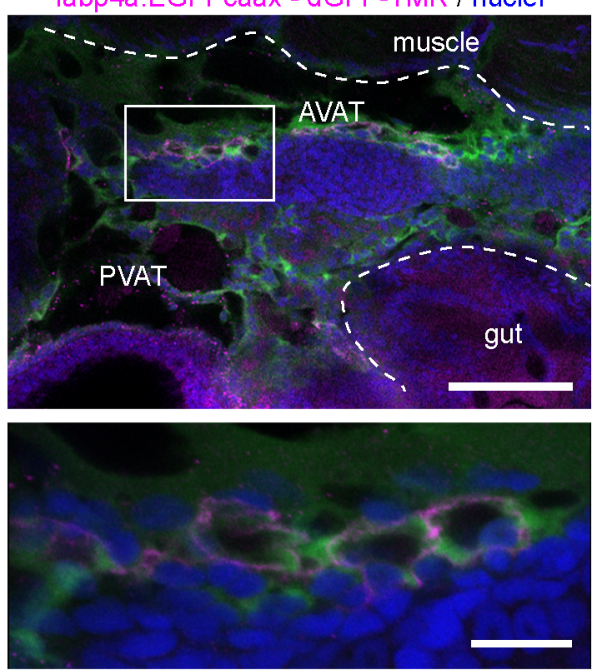

sense fabp4a probe-FITC / fabp4a:EGFPcaax - aGFP-TMR / nuclei
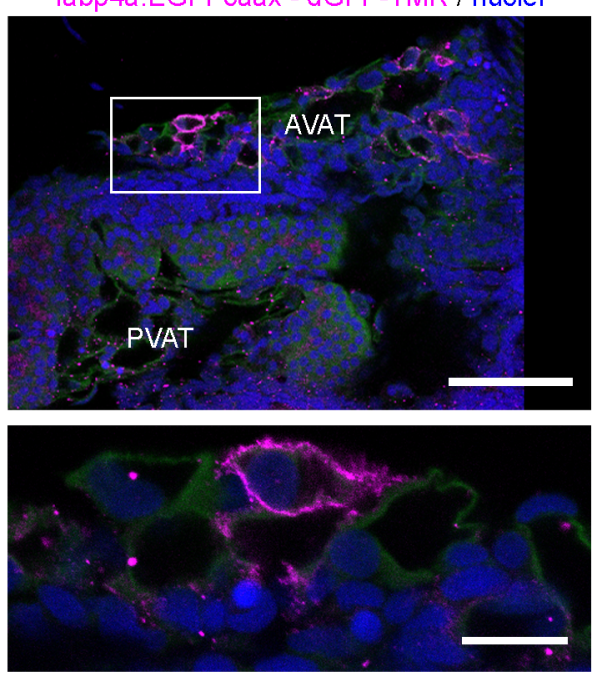

D

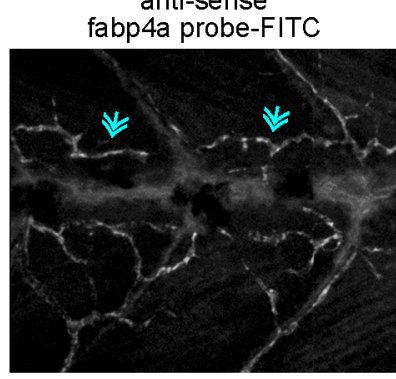

fabp4a:EGFPcaax aGFP-TMR

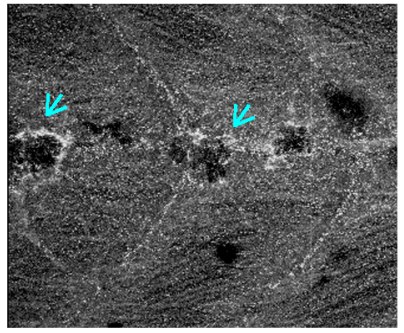
anti-sense fabp4a probe-FITC
fabp4a:EGFPcaax - aGFP-TMR

Fig. 2. Comparison of the expression pattern of fabp4a(-2.7):EGFPcaax and endogenous fabp4a mRNA in larva. Images of fabp4a(-2.7):EGFPcaax larvae of $21 \mathrm{dpf}$ processed for WMISH, immunolabeled with anti-GFP and analyzed in toto through confocal microscopy. (A) Panoramic (upper row) and magnified image (lower row) of the abdominal region of a larva labeled with fabp4a antisense probe. Yellow arrows denote the coincidence of EGFPcaax (immunofluorescence) and WMISH signal. Arrows with asterisks show regions with WMISH labeling and no EGFP signal. (B) Panoramic (upper row) and magnified image (lower row) of the abdominal region of a larva labeled with fabp4a sense probe. Yellow double arrows indicate regions with EGFPcaax signal without WMISH labeling. (C) Intensity profile of FITC fluorescence along the lines indicated in A and B insets. (D) Images of the trunk of a larva labeled with fabp4a antisense probe. Blue single arrows indicate pigment cells with EGFPcaax signal. Blue double arrows show WMISH labeling in blood vessels. Scale bars: A and B: $100 \mu \mathrm{m}$ (upper row), $20 \mu \mathrm{m}$ (lower row); D: $100 \mu \mathrm{m}$.

\section{Development of early adipocytes in vivo and their relationship with blood vessels}

Our results therefore supported the hypothesis that the fabp $4 a(-2.7)$ : EGFPcaax transgene marks adipocytes during differentiation. To test this possibility we analyzed live larvae and embryos of different stages using confocal microscopy. First, we analyzed embryos in search of early expression of fabp 4a(-2.7):EGFPcaax. In vivo confocal analysis of $2 \mathrm{dpf}$ and $5 \mathrm{dpf}$ embryos showed expression of 
EGFPcaax in cells along the antero-posterior axis at dorsal, lateral and ventral positions, all reminiscent of pigment cells (Fig. S3A,C, double arrows). The presence of early labeling of pigment cells suggested that this expression domain corresponded to ectopic expression of fabp 4a(-2.7):EGFPcaax in the transgenic embryos. To discard autofluorescence or dispersion of light by pigments, we immunostained fixed embryos with anti-GFP antibody. We observed immunostaining co-localizing with EGFPcaax fluorescence (Fig. S3B,D, double arrows), thus confirming that in our fish line pigment cells are labeled by fabp 4a(-2.7):EGFPcaax.

Previously, it has been reported that lipid droplets are first evident at the right side of the abdomen of early larvae, in ventral and posterior positions with respect to the swim bladder (Flynn et al., 2009; Minchin and Rawls, 2017b). Thus, we turned our attention into that region in larval stages and stained lipid droplets with LipidTOX-Red. Interestingly, early larvae of standard length (SL) $4.5 \mathrm{~mm}$ ( $8 \mathrm{dpf}$ ) showed labeling of small cells within the abdomen. At higher magnification we observed the expected surface localization of EGFP. However, these cells did not present lipid droplets (Fig. 3A). We also observed labeling of cells within the trunk in dorsal positions which corresponded to the presence of pigment cells in transmitted light images. Other signals in the images corresponded to autofluorescence of gut contents in the ventral-most part of the larvae and to pigment cells in the
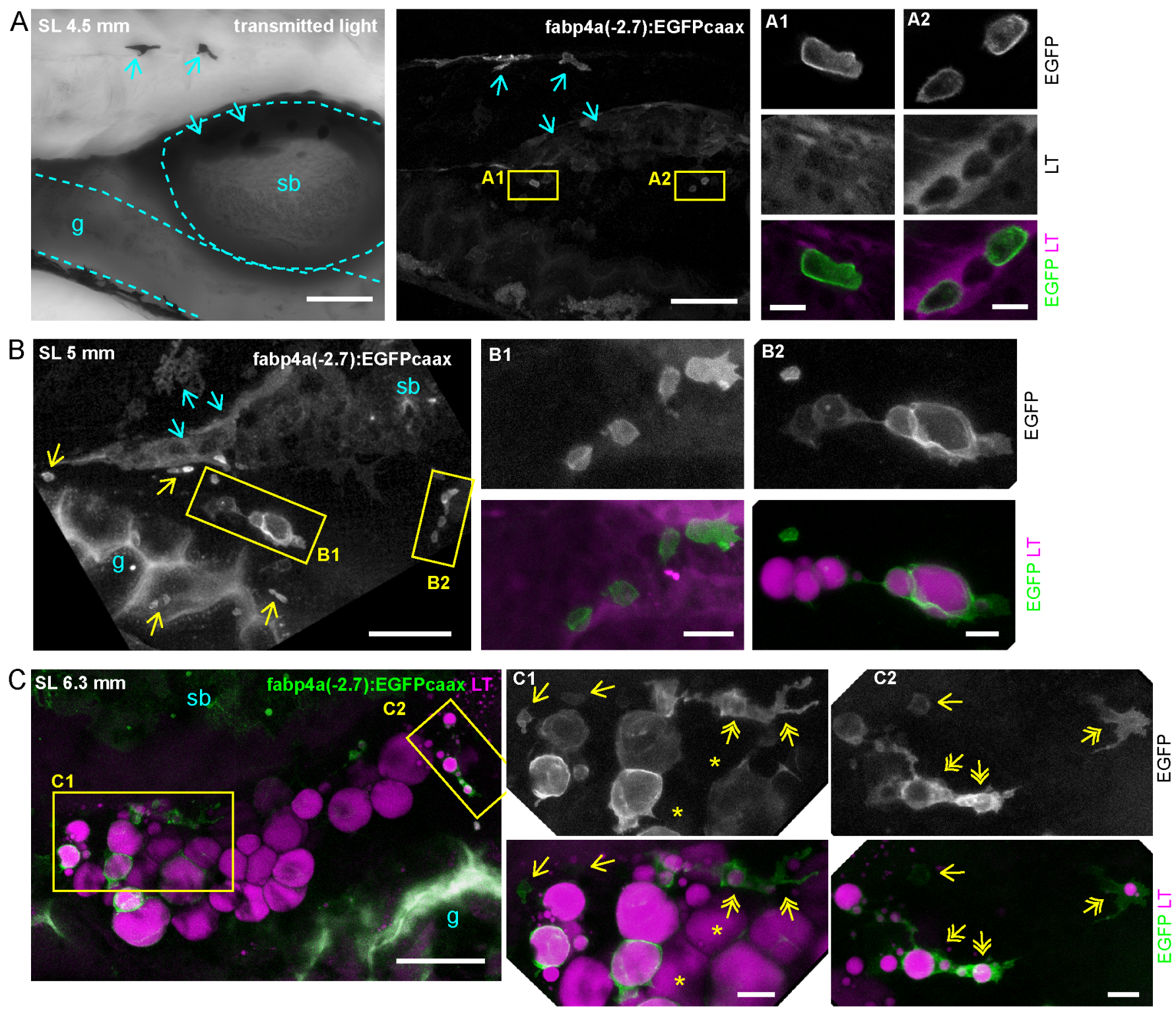

Fig. 3. Distribution of labeled cells in the abdominal region of live fabp4a(-2.7):EGFPcaax larva of different stages. Larvae of the indicated stages were stained with LipidTOX-Red, mounted in agarose and imaged using confocal microscopy. (A) Transmitted light and 3D projection images of a larva of SL $4.5 \mathrm{~mm}(8 \mathrm{dpf})$. Yellow rectangles denote cells with transgene labeling. Insets A1 and A2 show confocal sections of these regions. Note the membrane localization of EGFP and the lack of LipidTOX-Red labeling. Cyan arrows indicate pigment cells expressing the transgene. (B) 3D projection images of a larva of SL $5 \mathrm{~mm}$ (12 dpf). Yellow rectangles denote EGFP+ cells, magnified in B1 and B2. Cells with lipid droplets as well as without them (yellow arrows) can be seen in the same larva in different positions. (C) 3D projection images of a larva of SL $6.3 \mathrm{~mm}$ (16 dpf) with initial PVAT depot formation. Note the presence of EGFP+ cells with unique cell-filling lipid droplets, irregular cells with several lipid droplets (yellow double arrows) and small cells without lipid droplets (yellow single arrows). Asterisks indicate cells without EGFP expression. sb, swim bladder; g, gut. Scale bars: A: $100 \mu \mathrm{m}$ (panoramic view); $10 \mu \mathrm{m}$ (insets); B: $100 \mu \mathrm{m}$ (panoramic view); $20 \mu \mathrm{m}$ (insets); C: $100 \mu \mathrm{m}$ (panoramic view); $20 \mu \mathrm{m}$ (insets). 
dorsal half of the swim bladder (Fig. 3A). Despite taking several actions to diminish these confounding signals ( $16 \mathrm{~h}$ food restriction previous to imaging; incubation with epinephrine), they were persistent. Nevertheless, based on the surface localization and intensity of the EGFP signal it was possible to clearly distinguish these EGFPcaax positive and lipid droplet-free cells (EGFP+/LD-).

Next, we evaluated older larvae to assess whether these EGFP+ cells in the abdominal region could accumulate lipids. Importantly, larvae of SL 5 and $6.3 \mathrm{~mm}$ (12 and $16 \mathrm{dpf}$, respectively) clearly showed cells with surface EGFP signal and lipid droplets of various diameters (Fig. 3B,C). We also noted that some of these cells had irregular forms and projections (Fig. 3C, double arrows). Larvae of these ages also had rounder cells almost completely filled with one big lipid droplet (Fig. 3B,C). In mice, pre-adipocytes have been found to reside near blood vessels (Tang et al., 2008). Thus, we analyzed the relationship of early adipocytes with the vasculature by crossing fabp4a(-2.7):EGFPcaax fish with the kdlr:mCherry line, which labels endothelial cells. We observed EGFP+ cells both in close apposition and at some distance of vessels (Fig. 4A). Moreover, when cells with lipid accumulation were observed in the PVAT or AVAT depots, some usually appeared in close contact with vessels, sometimes with extensions surrounding them (Fig. 4B).

We expanded our analysis until $21 \mathrm{dpf}$ larvae (larvae of SL $7-$ $8 \mathrm{~mm}$ ) and observed EGFP+ positive cells with different morphologies (Fig. 5A,B). We observed rounded cells filled with a single lipid droplet, which likely correspond to mature adipocytes, in some cases having cell projections. Other cells, usually located at the periphery of the depots, typically showed one or more smaller lipid droplets and more irregular morphologies, also with membrane projections (Fig. 5A). In many occasions we observed cells in close proximity to vessels or with extensions surrounding them (Fig. 5B). Remarkably, in all stages analyzed in this work, EGFP+/LD- cells were present. These cells could be observed not only in the abdominal region in AVAT and PVAT but also surrounding the gut in different positions (Fig. 3B), including the cloaca region (Fig. S4). Interestingly, using transmitted light and high magnification we observed inclusions within EGFP+/LD- cells (Fig. 5C,D). We performed time lapse acquisitions of those cells for a short period of time. During these time lapse movies we observed that inclusions moved within the cytoplasm (Movie 1). Furthermore, we observed that cells could remain static or have directional movement over a cell diameter distance (Movie 1). This behavior was accompanied by the formation of protrusions that were also evident in our single time point observations (Figs 3B and 5C,D).

Altogether, our results show that fabp 4a(-2.7):EGFPcaax expressing cells are present in different larval stages, from just before the beginning of the accumulation of fat to later stages where lipid depots are readily visible. Furthermore, we found labeled cells with lipid droplets of different sizes, confirming that cells expressing fabp4a(-2.7):EGFPcaax in the abdominal region of larvae are adipocytes in different stages of differentiation. Even though our fish line also expresses EGFP in pigment cells, in vivo $3 \mathrm{D}$ analysis of the abdominal region efficiently allowed us to distinguish early and mature adipocytes based on localization and cell shape. Furthermore, the results underscore a tight relationship between adipocytes and vessels during their differentiation, and the coexistence of EGFP+ lipid-filled cells with EGFP+/LD- cells in the tissue.
Analysis of the lipid metabolic profile of early adipocytes with Nile Red fluorescence and spectral phasor plot analysis

As mentioned before, we hypothesized that EGFP+/LD- cells were in fact early adipocytes. Early adipocytes initiate lipid accumulation as part of their differentiation program, and thus would show a mixed lipid environment with neutral and polar components. The quantification of these components has been carried out before in cultured cells through Nile Red fluorescence analysis using spectral phasors (Di Giacinto et al., 2018; Maulucci et al., 2018). In our transgenic larvae, the fluorescence of EGFP could be used as a third component to identify the cells of interest (EGFP+ cells). Thus, we took advantage of the spectral phasor analysis to study the Nile Red spectral shift in the presence of EGFP fluorescence. A similar approach has been used to study membrane polarity using LAURDAN in the presence of mRuby fluorescence (Sameni et al., 2018). For the cellular lipid metabolic profile, wild-type larvae stained with Nile Red or fabp4a(-2.7):EGFPcaax larvae with or without staining with Nile Red were imaged using hyperspectral detection and the images were analyzed using the advantages of the model-free spectral phasors approach (Fig. S5, the analysis procedure is described in depth in the Material and Methods section) (Malacrida et al., 2017).

Wild-type larvae stained with Nile Red or fabp4a(-2.7): EGFPcaax larvae without staining were analyzed first to set the extremes of the distributions in the phasor plot (Fig. S5B). Notice that the Nile Red fluorescence was spread in a trajectory due to the heterogeneity in the polarity of Nile Red environments provided by the intracellular membranes. The position along the trajectory represents pixels corresponding to different fractions of membranes with more or less polarity. In the EGFP+ cells labeled with Nile Red, the linear combination for the Nile Red was dragged towards the EGFP position (Fig. S5C). Thus, the extremes of the Nile Red trajectory can be considered as two components and the EGFP as the third component. This strategy enabled us to generate masks for individual EGFP+ cells and to analyze their lipid polarity profile, avoiding the Nile Red signal from other cells. An example image is shown in Fig. S5C. Two cells, one with lipid droplets (cell A) and another without them (cell B), generated clusters at the phasor plot with unequivocally different distribution profiles. To analyze the lipid polarity profile on each of them, we obtained the polarity fractional plot (Fig. S5D). The analysis of cell A yielded a multimodal distribution with higher representation of intermediate zones, whereas cell B gave a single peak in the polar lipid region.

Using this approach, we analyzed EGFP+ cells in larvae at different stages. Representative examples of the observed profiles are shown in Fig. 6A and B. Seemingly mature adipocytes with a big lipid droplet showed a peak in Nile Red profile in regions corresponding to the accumulation of neutral lipids as expected (Fig. 6A,B, 'cell D'). Interestingly, it was possible to observe a small peak towards longer wavelengths, representing polar lipid components in the same cells, such as the plasma membrane. This was corroborated by the localization of these pixels: the former were localized centrally and the latter surrounded the whole cell (Fig. 6A, see Nile Red profile of 'cell D'). EGFP+/LD- cells usually showed distributions enriched in polar components (Fig. 6A,B, 'cell A'). Nevertheless, we imaged cells with several peaks or flatter distributions, probably representing transitions between polar and neutral lipid environments (Fig. 6A,B, 'cell B' and 'cell C'). To summarize and present all the observed profiles the center of mass $(\mathrm{CM})$ and distribution range (RD) of the lipid polarity profiles were calculated and used as characteristics of each distribution for comparison purposes (Materials and Methods; Fig. S5D). Within 

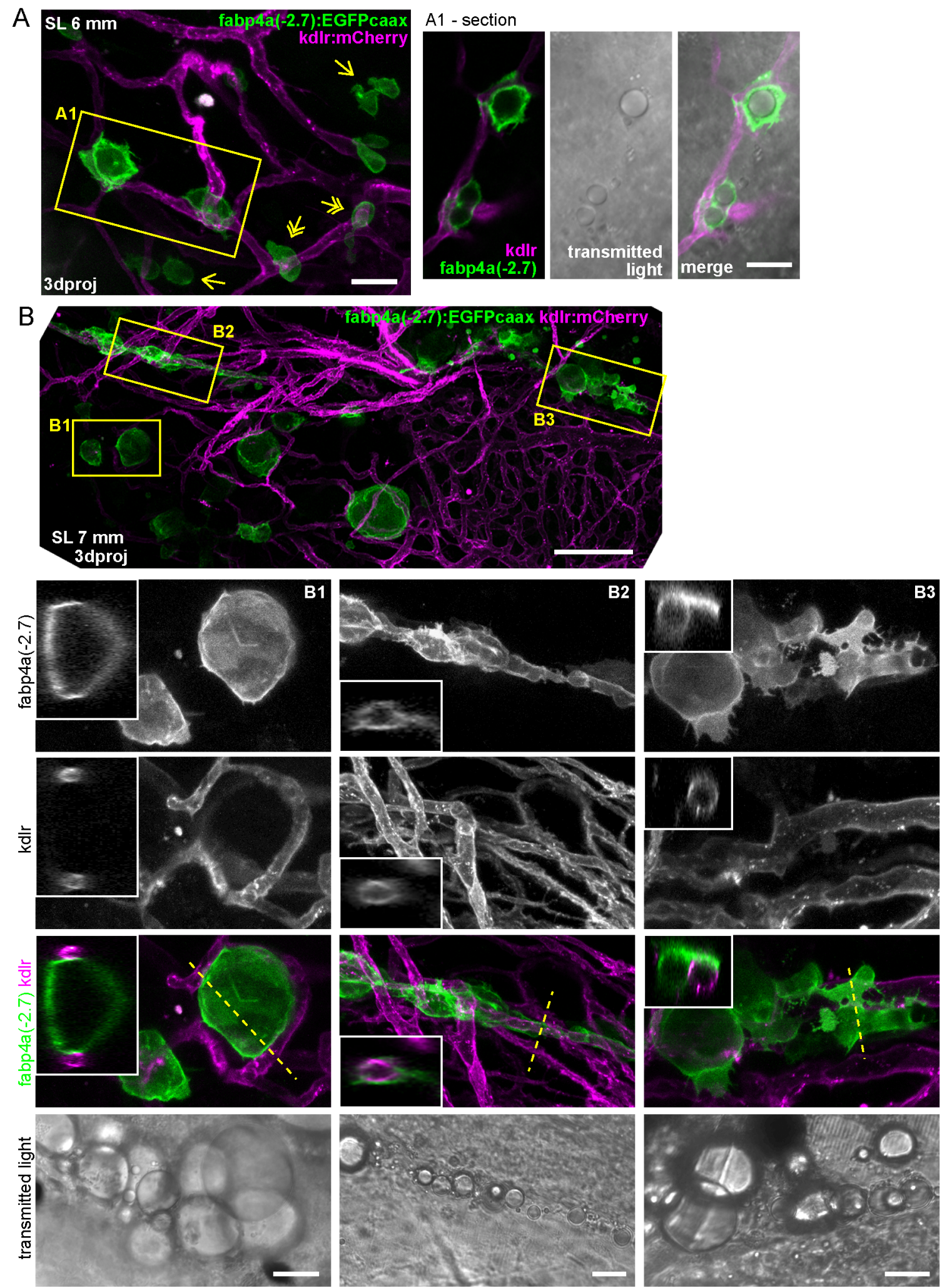

Fig. 4. Interaction of early adipocytes with blood vessels. Live larvae from the cross of fabp4a(-2.7):EGFPcaax and kdlr:mCherry fish lines were imaged through confocal microscopy. Images presented here are 3D projections or single sections, as indicated. (A) Larva of SL $6 \mathrm{~mm}$ (13 dpf) with many EGFP+ cells in its abdominal area, a few of them having lipid droplets (inset A1). Some of the cells are in contact with blood vessels (double arrows) and some of them are not (single arrows). (B) Larva of SL $7 \mathrm{~mm}$ (16 dpf), with PVAT and AVAT depots (only some cells of each depot expresses EGFP). Insets B1, B2 and B3 show EGFP+ cells with lipid droplets in close apposition to blood vessels and in some cases surrounding them (B2). 3D projections and sections through the position indicated by the dashed line are shown. Scale bars: A: $100 \mu \mathrm{m}$; B: $100 \mu \mathrm{m}$ (panoramic view), $20 \mu \mathrm{m}$ (insets). 
A
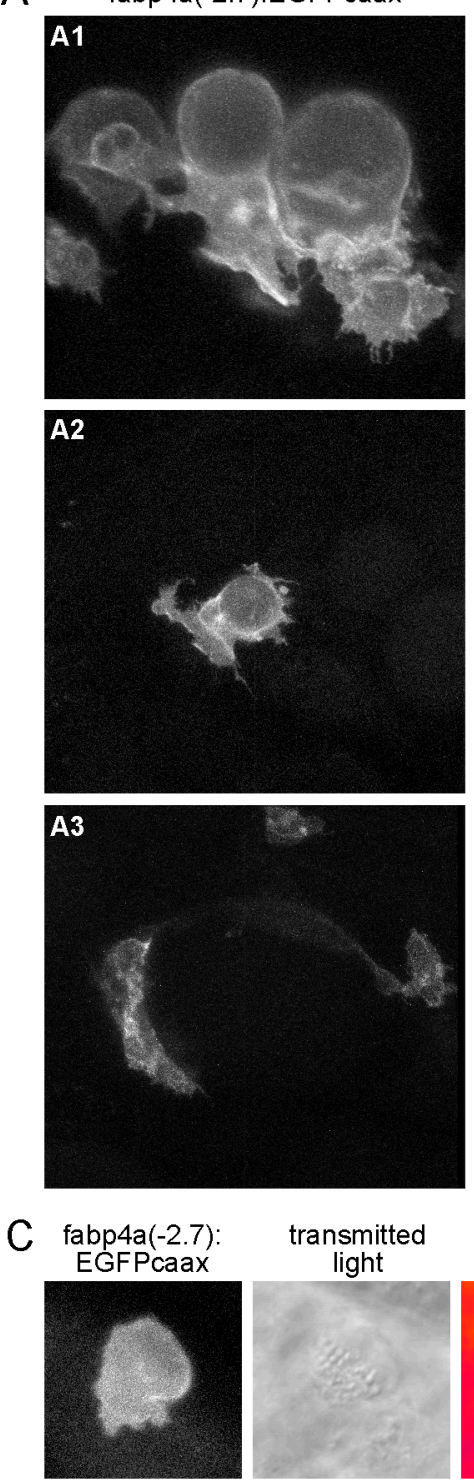

fabp4a(-2.7):EGFPcaax / LT
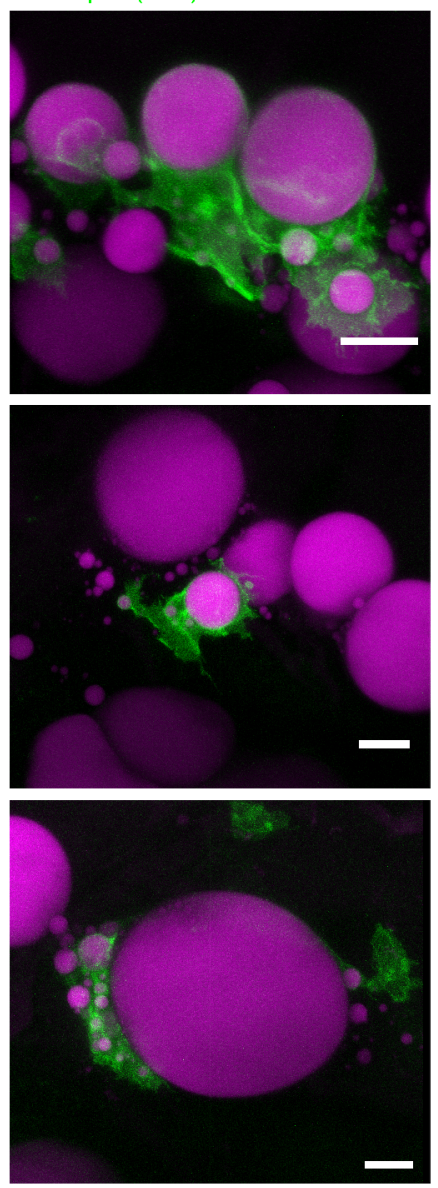

Nile Red

profile

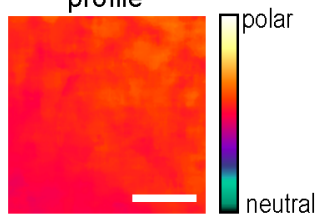

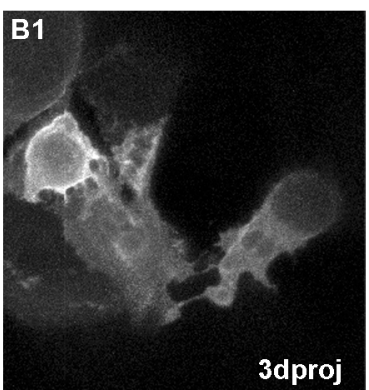
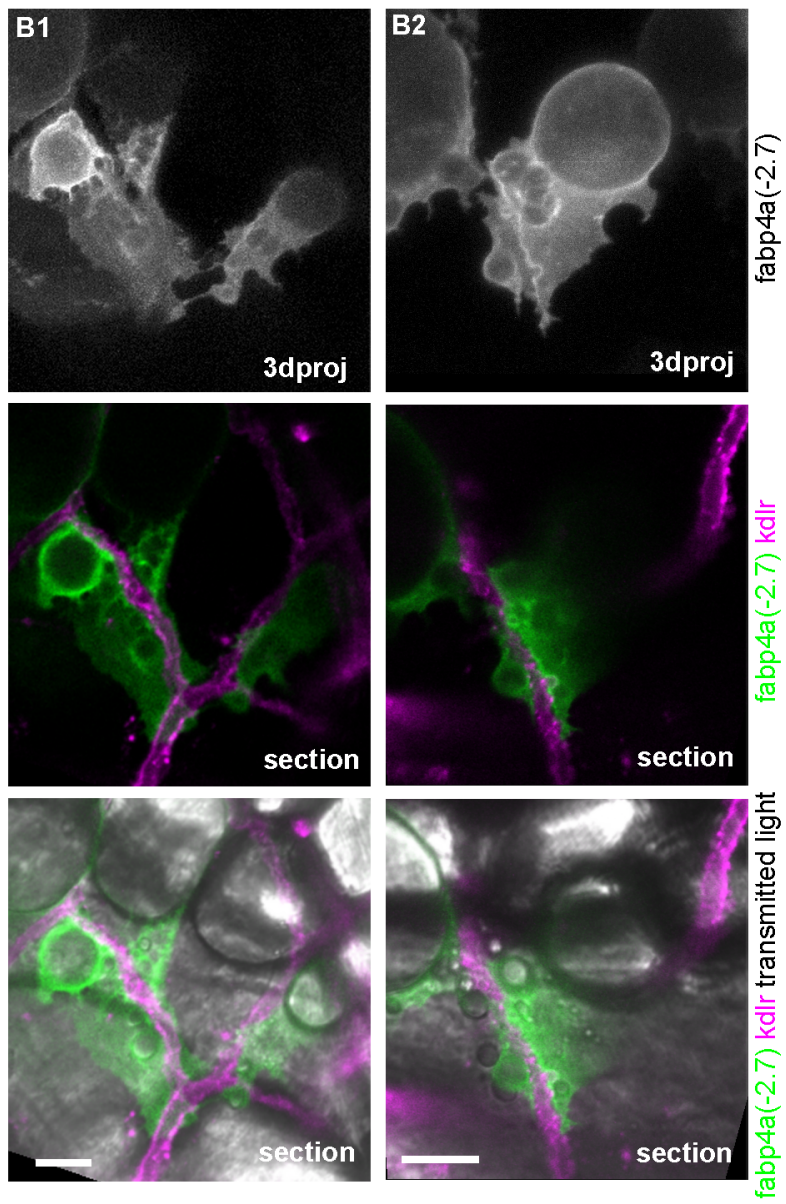

D

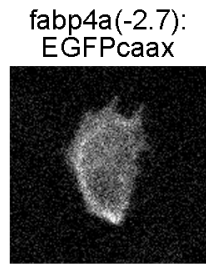

transmitted light

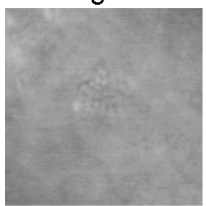

Fig. 5. Different cell morphologies observed in fabp4a(-2.7):EGFPcaax larvae. (A) fabp4a(-2.7):EGFPcaax larvae of 21 dpf were labeled with LipidTOXRed and imaged in vivo through confocal microscopy. Images are 3D projections of confocal stacks, to show different cell morphologies found in these larvae. (B) Images of fabp4a(-2.7):EGFPcaax; kdlr:mCherry larvae of SL $8 \mathrm{~mm}$ (19 dpf). Note labeled cells in the AVAT depot with cytoplasmic projections which lay in close apposition to blood vessels. Images are 3D projections or sections as indicated. (C,D) High magnification confocal sections of EGFP+ cells in larvae stained with Nile Red (C) or LipidTOX-Red (D). The cytoplasmic inclusions observed in transmitted light in these cells showed a polar lipid profile (evidenced by Nile Red staining, C) and low signal intensity of LipidTOX-Red (D). Scale bars: A: $20 \mu \mathrm{m} ; \mathrm{B}: 20 \mu \mathrm{m} ; \mathrm{C}, \mathrm{D}: 10 \mu \mathrm{m}$.

the plot of center of mass versus distribution range (Fig. 6C) it was possible to separate a subgroup of cells with statistically distinct median (for the center of mass and distribution range) and variability (only for the center of mass) compared to those cells outside this region (Fig. 6C,D, dashed line). The low $\mathrm{CM}$ and low DR means that the cells within this group were constituted mostly by polar lipids. These cells represented over $50 \%$ of the cells analyzed from 8 to $16 \mathrm{dpf}$ (Fig. 6E). The rest of the cells analyzed layed outside the low CM-low DR region due to increasing accumulation of neutral lipids, which extended the DR and biased the CM towards higher values. The percentage of cells analyzed that can be classified in this subgroup increased with larva age (Fig. 6E). Of note, we observed cells with distinct lipid polarity profiles among larvae of similar standard length and in some cases within the same larva (Fig. 6E). These observations implied the coexistence of adipocytes in different stages of differentiation within the same larva, suggesting that differentiation in vivo is continuous and asynchronous. These results indicate that our zebrafish fabp4a(-2.7):EGFPcaax transgenic line together with hyperspectral imaging and the spectral phasor analysis shown here is a powerful tool to study changes of the intracellular lipid environment in differentiating adipocytes in live zebrafish larvae.

\section{DISCUSSION}

A number of studies have started to address the role of compounds on lipid metabolism and storage using zebrafish as a model system (Landgraf et al., 2017). Current knowledge on zebrafish adipose tissue is restricted to fat accumulation capacity using lipophilic dyes due to the lack of specific cell markers. Here we generated and analyzed a new zebrafish line to specifically label adipocytes along their differentiation in vivo. 
A
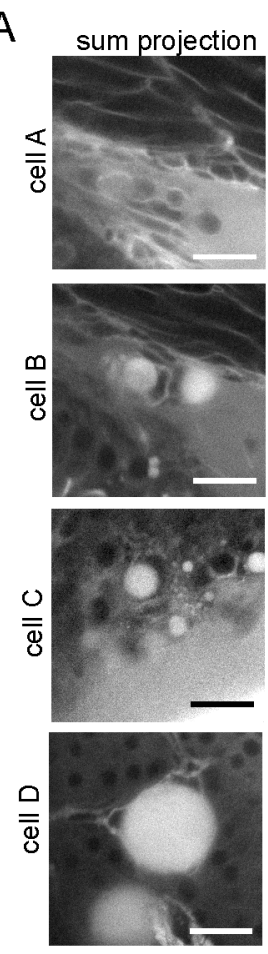
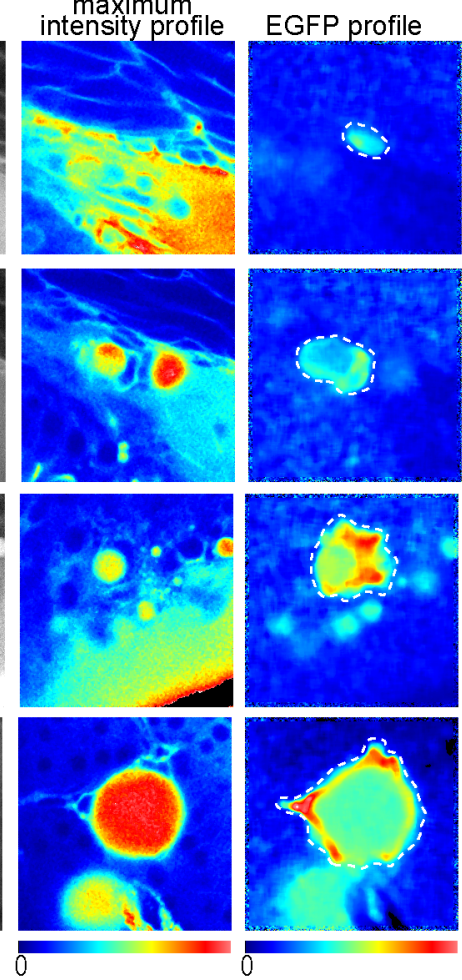
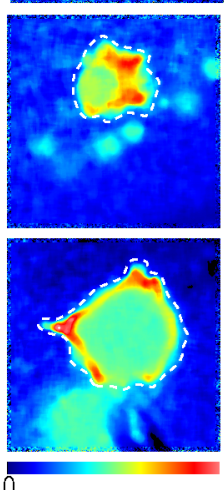
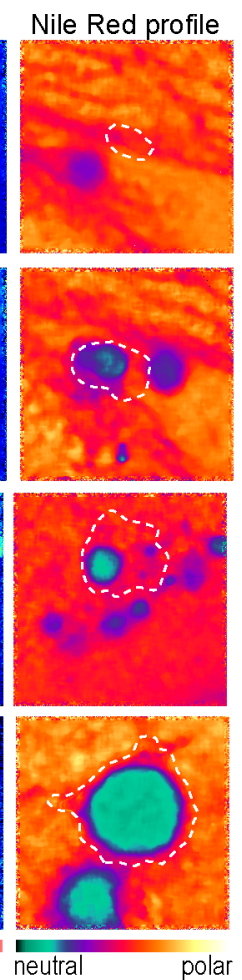

$\mathrm{E}$

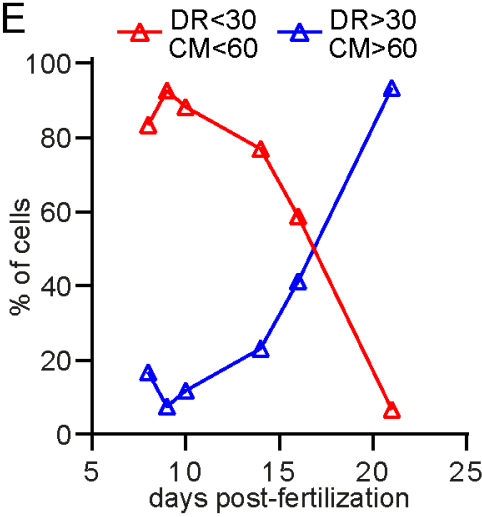

B
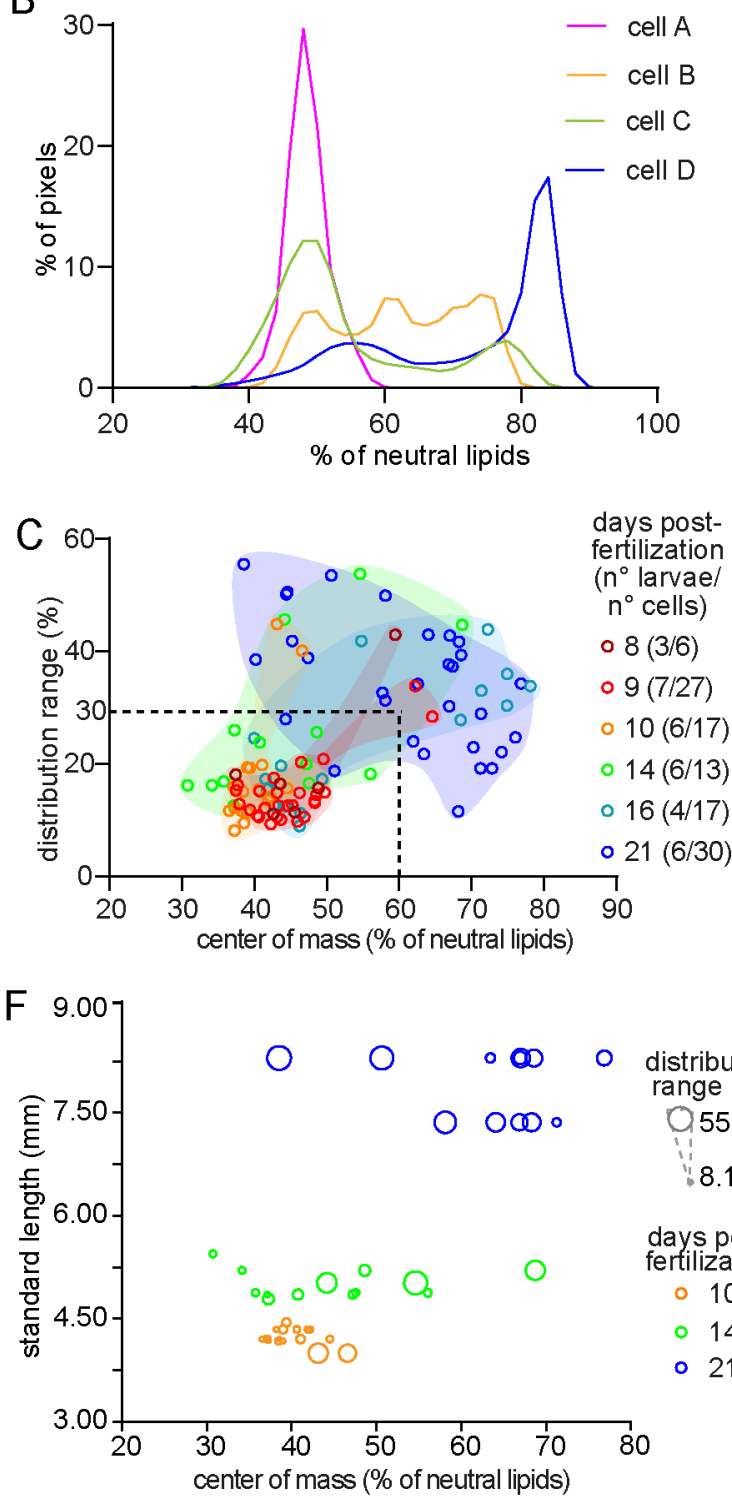

Fig. 6. Larvae in different stages present cells with distinct lipid metabolic profiles. (A) Representative hyperspectral images of adipocytes ('cell A' to 'cell D') in different stages of differentiation. Raw images are presented in gray and intensity based color scale. Images generated after phasor plot analysis make evident the EGFP and Nile Red profiles which are represented separately by different color scales. Scale bars: A: $20 \mu \mathrm{m}$. (B) Plot with the normalized distribution of the image pixels with respect to the fraction (expressed as percentage) of neutral lipids for each cell in A. (C) Scatter plot showing the distribution range and center of mass of the cells analyzed in different larval stages. Both variables are expressed as the percentage of neutral lipids. The group of data coming from the same larval stage were enclosed by a manually generated convex hull shape just for visualization purposes. The dashed lines $(D R=30 ; C M=60)$ generates the two regions considered in $D$ and $E$. The numbers in brackets indicate the total number of larvae and the total number of cells analyzed in each stage. (D) Data within $(\mathrm{DR}<30 / \mathrm{CM}<60)$ and outside $(\mathrm{DR}>30 / \mathrm{CM}>60)$ the region considered in $\mathrm{C}$ were separated and compared. For $\mathrm{CM}$ : $\left({ }^{*}\right)$ $P=2.06 \times 10^{-13}$ for median comparison (Mann-Whitney test), $P=1.62 \times 10^{-4}$ for coefficient of variation comparison (Fligner-Killeen test); $\left(^{* *}\right) P=2.24 \times 10^{-16}$ for median comparison (Mann-Whitney test), $P=7.1 \times 10^{-6}$ (Fligner-Killeen test). (E) Representation of the percentage of cells in each group (DR<30/CM $<60$ and $\mathrm{DR}>30 / \mathrm{CM}>60$ ) with respect to the larval stage presented in dpf. (F) Representation of the distribution range (as the size of the dots) and center of mass for the cells within some of the larva analyzed; each larva had a different standard length.

\section{Expression pattern of fabp4a(-2.7):EGFPcaax}

We generated a new transgenic line cloning the proximal part of the promoter of the lipid transporter gene fabp $4 a$ and used it to direct the expression of a membrane associated form of EGFP. It was shown previously that fabp $4 a$ is expressed in the lens, midbrain as well as in blood vessels in the head and trunk of $2 \mathrm{dpf}$ embryos (Liu et al., 2007), an expression pattern that we also observed with our WMISH assay in $2 \mathrm{dpf}$ embryos. However, the in vivo analysis of 2 and $5 \mathrm{dpf}$ embryos showed that fabp4a(-2.7):EGFPcaax transgene is not expressed in either blood vessels, midbrain or lens cells. In the case of $15 \mathrm{dpf}$ larvae, fabp $4 a$ mRNA was previously reported to be expressed in abdominal cells with or without neutral lipid accumulation as well as in trunk vessels (Flynn et al., 2009). In agreement with this report, our WMISH assay in larvae showed the expression of endogenous fabp $4 a$ in PVAT and AVAT depots as well as in blood vessels. However, the expression of fabp $4 a(-2.7)$ : EGFPcaax transgene colocalized with endogenous expression of fabp $4 a$ only in cells of AVAT and PVAT depots. Thus, the 
expression pattern of fabp4a(-2.7):EGFPcaax recapitulates primarily the adipose tissue domain of the endogenous expression pattern of fabp $4 a$. Interestingly, a recent report has shown that the $1.5 \mathrm{~kb}$ proximal region of fabp $4 a$ directing the expression of cytoplasmic EGFP, also results in an expression pattern restricted to adipose tissue (Mao et al., 2021).

Our fabp4a(-2.7):EGFPcaax transgene is expressed in pigment cells (melanophores and iridophores), a domain that does not coincide with the endogenous expression of fabp $4 a$. The presence of this expression domain may be due to the action of enhancers or repressors present in the genomic region where the transgene was integrated. This is a common drawback of using random insertion of transposons for transgenesis and highlights the importance of using complementary strategies such as insulators or targeted transgene insertion (Caldovic et al., 1999; Roberts et al., 2014). Importantly, however, the extra domain of expression in pigment cells did not hinder the utility of the transgenic line as it can be clearly separated from the adipose-related signal by considering the relative position of cells in 3D images.

Another particular aspect of this new transgenic fish line is that the level of expression of EGFP varies among cells within each larva. This variability remained even after three outcrosses with the wild-type fish line, discarding a mosaicism-based effect. Thus, variation among cells may reflect different cellular states along differentiation or, alternatively different subtypes of adipocytes. Progenitor and adipocyte subtypes with differential gene expression profiles within individual depots have been reported previously in mice and humans, probably reflecting their distinct developmental origins (revised in Luong et al., 2019). The promoter region used to generate the present transgenic line includes several binding sites for Ppar $\gamma$ and NF-K $\beta$ p50 that were able to modulate transcription in reporter assays (Laprairie et al., 2017). Moreover, Ppary has been shown to bind the fabp 4 promoter in a brown adipocyte cell line (Tontonoz et al., 1994). Also, it has been reported that fabp4 is regulated by VEGFA-DLL4/NOTCH and insulin-FOXO1 pathways in endothelial HUVEC cells (Harjes et al., 2014).

In humans, plasma levels of Fabp4, which is mainly produced by adipocytes, have been positively correlated with cardio-vascular disease, type-II diabetes and also with the progression of other diseases by a still undefined mechanism (Prentice et al., 2019). Thus, it would be interesting to analyze which factors contribute to the expression levels of fabp 4 as they may be of clinical relevance, and our results showing significant variability among adipocytes suggests this zebrafish line could be useful to this end.

\section{Characterization of early adipocytes and their relationship to blood vessels}

As mentioned, our detailed microscopic analysis of fabp $4 a(-2.7)$ : EGFPcaax larvae showed labeled cells with different characteristics. In the abdominal region, where WAT depots form first, we observed both EGFP+ cells with lipid droplets of various sizes and others without them. These observations are in agreement with previous reports showing that fabp $4 a$ is expressed in cells with and without lipid droplets in embryonic stages and early larvae (Flynn et al., 2009). Accordingly, we found EGFP+ cells in the ventral region of the tail and EGFP+/LD- cells in the abdominal region of early larvae (8-10 dpf) well before the initiation of fat accumulation. EGFP+/LD - cells were also observed in older larvae, coexisting with mature adipocytes in fat depots. Our analysis of Nile Red emission of EGFP+/LD- cells in the abdominal region revealed polar lipid or intermediate profiles. Thus, our results indicate that the fabp4a(-2.7):EGFPcaax transgene labels adipocytes in the PVAT and AVAT depots ranging from early stages of differentiation to mature differentiated cells. Future work will be focused towards analyzing the identity (pre-adipocyte versus early adipocyte) of EGFP+/LD- cells.

$\mathrm{EGFP}+/ \mathrm{LD}-$ cells in the abdominal region presented inclusions when observed with transmitted light at high magnification. These inclusions were LipidTOX-negative or showed a polar lipid profile in Nile Red hyperspectral analysis, and were highly motile within the cell when analyzed using time lapse acquisitions. Further experiments are required to determine the nature of these inclusions. One interesting possibility is that they may represent initial stages of lipid droplet formation in which the amount of accumulated neutral lipids is not enough to be observed through LipidTOX labeling. LD are formed through accumulation of neutral lipids within the lipid bilayer of the ER, initially forming structures denominated lenses which grow and bud becoming lipid droplets (Olzmann and Carvalho, 2019). Genetic labeling tools that have been developed to evidence initial neutral lipid accumulations may be implemented to study the conservation of early lipid droplet formation mechanisms in zebrafish (Kassan et al., 2013; Wang et al., 2016).

WAT progenitors expressing PPAR $\gamma$ have been reported to reside in the mural compartment of adipose blood vessels in mice (Hilgendorf et al., 2019; Tang et al., 2008). As an analogy to mammals, some authors have hypothesized that WAT progenitors in zebrafish may derive from perivascular pre-adipocytes or, alternatively, from hematopoietic tissue located in the caudal region (Salmerón, 2018). In double-labeled larvae, we found EGFP+/LD - cells both in contact and at a distance of blood vessels. In contrast, all EGFP+ cells with lipid droplets were observed in contact with blood vessels. EGFP+/LD- cells were also present surrounding the gut at different positions along the antero-posterior axis. Furthermore, our time lapse acquisitions revealed that these cells had the capacity to migrate. Thus, our results are consistent with the previous formulated hypothesis and in vivo time lapse microscopy of EGFP+ cells combined with cell tracing may provide further information. For this, new methods to maintain larvae alive through extended periods of time will be needed, since in our hands, larvae remained alive only for a few hours after mounting in agarose.

Our work also provides information about adipocytes during differentiation and in their mature state. As our transgenic approach included a membrane associated form of EGFP, we could clearly identify the presence of membrane protrusions in early and mature adipocytes. In double-labeled larvae we could appreciate that these membrane protrusions reached blood vessels, suggesting the presence of physical connections. Whether this interaction is direct between cell membranes or indirect through ECM components remains to be determined. Evidence exists in favor of the latter hypothesis, showing that modification of extracellular matrix composition by endothelial cells directs the hyperplasia or hypertrophy of adipocytes (Minchin et al., 2015). Alternatively, extensive evidence supports that several soluble factors coordinate adipogenesis and angiogenesis in obesity as well as in adiposederived stem cell therapy (Hutchings et al., 2020; Lemoine et al., 2013). Furthermore, secretion of factors by peri-arterial adipocytes can mediate protection or inflammation of the adventitia and atherosclerosis development (Kim et al., 2020). Much less information is available on the interaction of adipocytes and vessels during formation of the adipose tissue (Cao, 2007). Our results suggest an intimate relationship of early adipocytes with blood vessels, probably through cell surface molecules. We hypothesize that these interactions may be instrumental in the 
acquisition of lipids from blood vessels as well as in regulating adipose depot growth.

\section{Nile Red and phasor approach to characterize in vivo cell lipid metabolism}

We used the new fabp4a(-2.7):EGFPcaax line to implement a tool for in vivo analysis of lipid environment using Nile Red hyperspectral imaging and its analysis through spectral phasor plots (Maulucci et al., 2018). Early adipocytes initiate lipid accumulation as part of their differentiation program, and thus would show a mixed lipid environment with neutral and polar components in their profile. Indeed, as mentioned before, EGFP+/LD- cells showed different profiles, ranging from polar-lipid environments to intermediate polarity-lipid environments. Several groups have studied the lipid composition of in vitro differentiating adipocytes of different origins through disruptive methods (Miehle et al., 2020). For example, human undifferentiated adipocytes were enriched in membrane phospholipids such as phosphatidylethanolamines, phosphatidylcholines and sphingomyelins. Meanwhile, completely differentiated cells were shown to present diacylglycerols, lysophosphatidylethanolamines and triacylglycerols in addition to membrane phospholipids. Thus our results are consistent with previous analyses, and importantly, provide a base to build on the metabolic analysis of individual cells in their natural context.

Our data show that the technique is sensitive enough to detect lipid environment changes in a non-invasive way and for a specific cell identity, opening the possibility of using this tool to evaluate the progression of differentiation in vivo or the effect of drugs on lipid metabolism or genetic interventions. Future development of other fish lines using earlier molecular markers will improve the observation of cells in different stages. For example, work in mice have used prefl and $z f p 423$ to mark adipose tissue progenitors and pre-adipocytes (Gupta et al., 2010; Hudak et al., 2014). Both of these genes are present in zebrafish and may be useful to track the origin of the adipocyte lineage.

\section{Conclusion}

In this work we introduced a new zebrafish line labeling adipocytes from early stages up to fully differentiated cells. Furthermore, we described the interaction of early and differentiated adipocytes with blood vessels and evidenced early lipid metabolic changes in vivo. We anticipate that the new transgenic line described here will be a useful tool to study the cell biology of adipocytes in the context of the tissue and the whole organism, their interaction with blood vessels and their differentiation in vivo. Recently, new fish lines labeling lipid droplets have been generated (Lumaquin et al., 2021; Wilson et al., 2021 preprint) that may be combined with the fabp4a(-2.7):EGFPcaax line presented here for screening approaches focused on genetic and environmental factors affecting early adipocyte differentiation. The fabp $4 a(-2.7)$ :
EGFPcaax fish lines and the genetic tools available in zebrafish, combined with two-photon and multiplexing microscopy will surely provide a powerful platform to gain in depth information on adipogenesis and its in vivo determinants.

\section{MATERIALS AND METHODS}

\section{Zebrafish maintenance and growth}

We worked with $\mathrm{AB} / \mathrm{TU}$ (wild-type fish line), $\mathrm{Tg}($ kdlr:mCherry)is5 (blood vessel labeling; Wang et al., 2010) and $\operatorname{Tg}($ fli1:EGFP)yl (blood vessel labeling; Lawson and Weinstein, 2002). Danio rerio adults were maintained in a stand-alone system (Tecniplast), at $28^{\circ} \mathrm{C}, 800 \mu \mathrm{S} / \mathrm{cm}^{2}$, and $\mathrm{pH} 7.5$, with a diet based on live $48 \mathrm{~h}$-post eclosion Artemia salina (artemia cyst from Artemia International) and pellet (TetraMin, tropical flakes, Tetra). Embryos were raised in Petri dishes with aquarium water at $28.5^{\circ} \mathrm{C}(50$ larvae per $10 \mathrm{~cm}$ Petri dish) and bleached at $24 \mathrm{~h}$ post-fertilization. For growth of larvae we used Larval AP100-1 $(<50 \mu \mathrm{m}$; Zeigler $)$ from 5 to $30 \mathrm{dpf}$ and Golden Pearl Reef \& Larval Diet (100-200 $\mu \mathrm{m}$; Brine Shrimp Direct) from 15 to $30 \mathrm{dpf}$. Dry food were administered twice per day plus one extra feed of live 24 h-post hatching A. salina.

Embryonic staging was performed according to Kimmel (Kimmel et al., 1995) up to $5 \mathrm{dpf}$ and larvae staging (after $5 \mathrm{dpf}$ ) was done according to Parichy (Parichy et al., 2009). SL is the distance between the tip of the nose and the caudal peduncle, and it correlates linearly with the growth of adipose tissue as well as the development of other characteristics in larval zebrafish (Minchin and Rawls, 2017b). All protocols ( $n^{\circ} 007-19,009-19,010-19$, 011-19) were approved by the Institut Pasteur de Montevideo ethics committee for the use of animal models (CEUA) and performed by trained, certified staff.

\section{Promoter cloning}

For identification of the potential promoter regions we combined manual analysis and a trial version of Gene2Promoter software (Genomatix). We then designed primers using the Primer-Blast tool from NCBI (Table 1). Candidate primers were blasted against the whole zebrafish genome using the BLAT tool from UCSC Genome Browser. Restriction sites were added at their $5^{\prime}$ end to enable directional cloning (underlined in Table 1).

We used zebrafish high-molecular weight genomic DNA, extracted as previously described (Sambrook et al., 1990) from $48 \mathrm{hpf}$ TAB embryos. For $f a b p 4 a$, the BAC DKEY-241P5 (Source BioScience) was used as a template. Each region was first cloned into pCRII plasmid using TOPO-TA Cloning Kit (Thermo Fisher Scientific). After sequencing, they were subcloned into p5'Entry-MCS plasmid from Tol2 Kit (Kwan et al., 2007) through digestion and ligation with T4 ligase (Thermo Fisher Scientific). We recombined each $\mathrm{p} 5^{\prime}$ Entry vector with pMiddle Entry vector coding for EGFPcaax (caax is a prenylation signal, directing EGFP to the plasma membrane), p3'Entry Vector with poly-A signal and the pDestTol2-CG2 backbone (with cardiac myosin light chain promoter directing the expression of GFP, cmlc2:GFP; and tol2 sites for insertion into the genome).

\section{Transgenic line generation}

TAB5 embryos at one cell stage were injected with 10-20 pg of the desired vector plus 10-20 pg of Tol2 Transposase mRNA. We then selected $24 \mathrm{hpf}$ embryos showing GFP fluorescence in the heart. We grew these embryos

Table 1. Primers used for amplification of the selected promoter regions, its position in relation to the transcription start site and the size of the amplification product

\begin{tabular}{|c|c|c|c|c|}
\hline Gene & Forward primer & Reverse primer & $\begin{array}{l}\text { transcription start } \\
\text { site }\end{array}$ & $\begin{array}{l}\text { Size } \\
\text { (bp) }\end{array}$ \\
\hline$c f d$ & 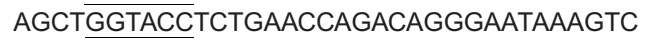 & ACGTGGATCCGTGTGCTTTAGCCTCTTGCC & -1032 to +234 & 1266 \\
\hline cebp $\alpha$ & ACGTGTCGACTCCGCTCGGGTAAATAAAGA & ACGTGGATCCAGCAACCTGTCGTGACTGTG & -1903 to +107 & 2010 \\
\hline
\end{tabular}

The underlined regions correspond to the restriction enzyme site. 
until 15-21 dpf when we analyzed the presence of fluorescence in adipose tissue. Selected individuals were outcrossed with the wild-type line until the third generation.

\section{Fixation, permeabilization and immunolabeling}

To decrease pigmentation, embryos were treated with PTU $0.3 \%$ starting at $8 \mathrm{hpf}$. For the same purpose larvae were anesthetized using tricaine $0.04 \mathrm{~g} / \mathrm{l}$, incubated in epinephrine $10 \mathrm{mg} / \mathrm{ml}$ plus tricaine $0.04 \mathrm{~g} / \mathrm{l}$, mounted in methylcellulose and observed using the stereomicroscope to select larvae expressing GFP. Fixation was carried out in 4\% PFA in PBS overnight at $4^{\circ} \mathrm{C}$.

Fixed embryos and larvae were permeabilized and immunolabeled following the protocol described by Inoue and Wittbrodt with minor modifications (Inoue and Wittbrodt, 2011). Briefly, all steps were carried out at room temperature with agitation unless stated otherwise. Fixed embryos and larvae were washed in PBS plus 1\% Triton X100 (PBST) $(3 \times 10 \mathrm{~min})$, dehydrated in a methanol series $(50: 50$ and 100:0 methanol: PBST) $\left(1 \times 10 \mathrm{~min}\right.$ each) and incubated in $100 \%$ methanol at $-20^{\circ} \mathrm{C}$ for $20 \mathrm{~min}$. After rehydration in the same methanol series, we performed an antigen retrieval step with $150 \mathrm{mM}$ Tris- $\mathrm{HCl} \mathrm{pH} 9$ (5 min at RT and $15 \mathrm{~min}$ at $\left.70^{\circ} \mathrm{C}\right)$. After a wash step in PBST $(10 \mathrm{~min})$ and two washes in distilled water (5 min each) we further permeabilized samples incubating them in $100 \%$ acetone at $-20^{\circ} \mathrm{C}$ for $20 \mathrm{~min}$. Finally we washed the samples in PBST several times $(6 \times 5 \mathrm{~min}$ each). For immunofluorescence on WMISH embryos and larvae we followed the same protocol without the acetone permeabilization step.

For immunolabeling all steps were performed with agitation. We incubated permeabilized embryos and larvae in the blocking buffer $(10 \%$ FBS plus $1 \%$ BSA in PBST) for $1 \mathrm{~h}$ at room temperature (RT). Primary and secondary antibodies were diluted in the incubation buffer (1\% FBS plus $1 \%$ BSA in PBST). Antibody incubations were performed at $4^{\circ} \mathrm{C}$ for 3 days, and washes at RT with PBST. The antibodies used in this work were: antiGFP (Invitrogen \#A11122, 1/500), anti-rabbit-633 (Invitrogen \#A21070, $1 / 1000)$.

\section{WMISH}

We cloned a region of $f a b p 4 a$ previously used for probe generation (Flynn et al., 2009) using the following primers: fwd: GATCAAATCTCAATTTACAGCTGTTG; rv: TTCAAAGCACCATAAAGACTGATAAT and oligodT retro-transcribed cDNA as a template. The amplified region was ligated into pGEM T-easy vector (Thermo Fisher Scientific). Selected clones were checked through digestion and sequencing. The selected clone has the region 195 to 648 from fabp $4 a$ mRNA sequence, spanning the 3 half of the CDS and part of the $3^{\prime} \mathrm{UTR}$, flanked by T7 and SP6 promoters in $5^{\prime}$ and $3^{\prime}$, respectively. To synthesize the probes we amplified the template using T7 and SP6 primers and afterwards generated digoxigenin (DIG) labeled probes by in vitro transcription with T7 or SP6 polymerases, using Digoxigenin-11-UTP (Merck). As an additional specificity control we used a slit 2 antisense probe which has already been tested (generously provided by C. Davison, Facultad de Ciencias, Universidad de la República; Davison and Zolessi, 2021)

The WMISH technique was performed as previously described (Koziol et al., 2014) with modifications following Flynn et al. and Elizondo et al. (Elizondo et al., 2005; Flynn et al., 2009). A detailed protocol is available upon request. Briefly, embryos and larvae were fixed in 4\% PFA prepared in PBS-DEPC water overnight at $4^{\circ} \mathrm{C}$. PFA was then replaced twice with $100 \%$ methanol and samples were stored at $-20^{\circ} \mathrm{C}$ until used. After rehydration in an ethanol series, larvae were permeabilized with $15 \mu \mathrm{g} / \mathrm{ml}$ Proteinase K (Fermentas) in PBS-0.1\% Tween-20 (PBS-T) for 10 min (for embryos) or $30 \mathrm{~min}$ (for larvae) at RT. After a rinse with triethanolamine buffer $(0.1 \mathrm{M}$, $\mathrm{pH} 8)$, they were treated twice with acetic anhydride $(0.25 \% \mathrm{v} / \mathrm{v}$ for $5 \mathrm{~min}$ each), washed with PBS-T, refixed with 4\% PFA in PBS-T for 20 min and washed extensively with PBS-T at RT. Pre-hybridization was performed overnight at $60^{\circ} \mathrm{C}$ in hybridization buffer $(50 \%$ formamide, $5 \mathrm{X} \mathrm{SSC}, 1 \mathrm{mg}$ / $\mathrm{ml}$ Torula RNA, $100 \mu \mathrm{g} / \mathrm{ml}$ heparin, 1x Denhardt's solution, $0.1 \%$ Tween$20,0.1 \%$ CHAPS, DEPC treated water). DIG-labeled probes were denatured at $80^{\circ} \mathrm{C}$ for $3 \mathrm{~min}$ and diluted to $0.2 \mathrm{ng} / \mu \mathrm{l}$ in the hybridization buffer. Hybridization was performed at $58^{\circ} \mathrm{C}$ for 2 days with agitation. Washing steps were done in hybridization buffer at $58^{\circ} \mathrm{C}$ with agitation, twice for 10 min each, then three times with $2 \mathrm{X} \mathrm{SSC}$ plus $0.1 \%$ Tween- 20 at $58^{\circ} \mathrm{C}$ for $20 \mathrm{~min}$ each, three times with $0.2 \mathrm{X}$ SSC plus $0.1 \%$ Tween- 20 at $58^{\circ} \mathrm{C}$ for $30 \mathrm{~min}$ each, and finally twice with maleic acid buffer (MAB) at RT for $15 \mathrm{~min}$. Samples were then blocked overnight at $4^{\circ} \mathrm{C}$ in $1 \%$ blocking reagent (Roche) plus 5\% sheep serum in MAB and incubated with anti-DIG conjugated to Peroxidase (1/50; Merck \#11207733910 Roche) in 1\% blocking reagent diluted in $\mathrm{MAB}$ for 3 days at $4^{\circ} \mathrm{C}$. Washing steps were done in MAB (three washes of $5 \mathrm{~min}$, followed by three washes of $1 \mathrm{~h}$ ), Fluorophore deposition was carried out with fluorescein-tyramide, prepared and developed as described by Hopman et al. (1998). After washes in PBS-T, samples were stored in $80 \%$ glycerol in PBS at $-20^{\circ} \mathrm{C}$ until used.

\section{In vivo labeling and imaging}

For in vivo lipid labeling, selected larvae were incubated in a $10 \mathrm{~cm}$ Petri dish (when labeled in group) or 12-well plate (when labeled individually) containing the lipophilic dye diluted in system water. We incubated the larvae with LipidTox Red (Invitrogen, 1/5000) for $1 \mathrm{~h}$ at $28^{\circ} \mathrm{C}$ or Nile Red (Sigma-Aldrich, $0.78 \mu \mathrm{M}$ for adipose area quantification and $0.078 \mu \mathrm{M}$ for emission spectra analysis) for $1 \mathrm{~h}$ at $28^{\circ} \mathrm{C}$. Labeled individuals were anesthetized and incubated in epinephrine as described above and mounted in $0.8 \%$ low melting point agarose in a $3.5 \mathrm{~mm}$ glass bottom Petri dish. After solidification, the sample was covered with tricaine $0.04 \mathrm{~g} / 1$ in system water. To ensure viability during the observation period, a block of agarose covering the region of the gills and the lower jaw was removed using a needle.

Low magnification images were acquired using a stereoscope (Nikon SMZ-445) with a canon EOS T3i-Rebel camera. More detailed in vivo images were acquired using epifluorescence or confocal microscopy. For epifluorescence we used an Olympus IX81 with 10x UPlan FLN 0.3 NA and 20x UPlan FLN 0.5 NA Olympus objectives. Confocal microscopy images were acquired with either a Zeiss LSM 800 or Zeiss LSM 880 with a $25 \mathrm{x}$ LD LCI Plan-Apochromat 0.8 NA Imm Corr DIC M27 (glycerol, oil, water, silicone) Zeiss objective. Hyperspectral imaging of Nile Red fluorescence was done using the lambda module in the Zeiss LSM 880 with the $25 \mathrm{x}$ objective, excitation the 488 Argon laser line was used and the spectra acquisition involved 22 step with $10 \mathrm{~nm}$ bandwidth (from $493 \mathrm{~nm}$ to $713 \mathrm{~nm}$ ) using a PMT-GaAsP detector.

\section{Image analysis}

Brightness-contrast adjustments were done using Fiji software (Schindelin et al., 2012). Fluorescence profile of MWISH images were generated using the Intensity Toolset from Imperial College of London FILM facility (https://www.imperial.ac.uk/medicine/facility-for-imaging-by-lightmicroscopy/software/fiji/).

For Nile Red hyperspectral data analysis we used the spectra phasor approach using Globals for Images SimFCS 4 software (G-SOFT Inc., Champaign, IL, USA). This method transforms the spectral data in each pixel to the real and imaginary component of the Fourier transform, as described earlier by Malacrida et al. (2016):

$$
\begin{aligned}
& G(\lambda)=\frac{\int_{\lambda \min }^{\lambda \max } I(\lambda) \cos (2 \pi n(\lambda-\lambda i) / \lambda \max -\lambda \min ) d \lambda}{\int_{\lambda \min }^{\lambda \max } I(\lambda) d \lambda}, \\
& S(\lambda)=\frac{\int_{\lambda \min }^{\lambda \max } I(\lambda) \sin (2 \pi n(\lambda-\lambda i) / \lambda \max -\lambda \min ) d \lambda}{\int_{\lambda \min }^{\lambda \max } I(\lambda) d \lambda} .
\end{aligned}
$$

$\mathrm{I}(\lambda)$ is the intensity at each step, $n$ the harmonic number and $\lambda_{i}$ is the initia wavelength. Each pixel in the image will be located at a single $(\mathrm{G}, \mathrm{S})$ position at the spectral phasor plot, yielding a cluster of points due to all pixels in an image. This transformation does not modify the original data and does not involve any fitting or any assumption of components. The position at the phasor depends on the spectrum maximum (phase angle, $\Theta$ ) and the full width at half maximum (Modulation, M) (Fig. S5A), as:

$$
\begin{aligned}
& M=\sqrt{S^{2}+G^{2}}, \\
& \theta=\arctan (S / G) .
\end{aligned}
$$


While red spectral shift implies increasing phase angle, the band narrowing moves the position toward the spectral phasor perimeter (modulation increases)

The spectral phasor plot enables the use of vector properties, such as the linear combination and the reciprocity principle. The linear combination allows the quantification of multiple components in a mixture as a sum fractions of single emitters. In our experiments, Nile Red presented complex photophysics that involved the emission from polar and neutral environments (membrane and lipid droplets, respectively). Furthermore, our phasor plots had an extra component from expression of EGFP. Using the three-component analysis developed by Ranjit and collaborators, we decomposed the fraction of Nile Red in the pixels with EGFP signal (Ranjit et al., 2019). We defined two individual cursor positions (two of the vertices) from the Nile Red trajectory extremes using images from wild-type larvae labeled with Nile Red, and the third position using images of unlabeled fabp 4a(-2.7):EGFPcaax larvae. The reciprocity principle enables to trace back a region of interest from the spectral phasor (imaginary space) to the original image (real space; the opposite, from a segmentation in the real image to the phasor plot, is also possible). Using this property, we segmented individual cells selecting the corresponding pixels in the phasor plot. Then, we obtained the fractional contributions for the Nile Red trajectory as explained in detail elsewhere (Ranjit et al., 2019). For comparison purposes between different treatments we used the CM for the Nile Red fraction histogram as a central tendency value and the range of the distribution as a dispersion value. The $\mathrm{CM}$ for the distribution of each cell Nile Red fraction was calculated as:

$$
\sum b . f(x) / \sum f(x)
$$

with ' $b$ ' being the percentage of pixels at the particular fraction ' $f(x)$ ' of the component ' $x$ ' (Malacrida and Gratton, 2018). The range of the distribution or distribution range (DR) was considered as the $f(x)$ interval that contains $96 \%$ of the pixels. For its calculation we used the accumulated distribution for $f(x)$ and determined the difference between the $f(x)$ values corresponding to $2 \%$ and $98 \%$ of the accumulated distribution.

\section{Statistical analysis}

The statistical analysis was performed using PAST software (Hammer et al., 2001) or Real Statistics Resource Pack software [Release 7.6, Copyright (2013-2021), Charles Zaiontz, www.real-statistics.com, accessed on March 2021]. For group comparisons we analyzed normality using Shapiro-Wilk test and homogeneity of variances using Levene test. Non-normal and homoscedastic distributions were compared with non-parametric tests (Kruskal-Wallis or Mann-Whitney with Bonferroni correction) as indicated in each case. Non-normal and heteroscedastic samples were rank transformed (Conover and Iman, 1981) and compared using Welch test and Games-Howell post-hoc test. For the comparison of coefficient of variation we used the Fligner-Killeen test.

\section{Acknowledgements}

We thank Luisa Berná for her help defining the putative promoter regions of our genes of interest and Hugo Naya for his advice on statistical analysis. We also thank Gisell Gonzales for her invaluable support in the zebrafish laboratory as well as Magdalena Cardenas and Flavio Zolessi for the revision and helpful comments about the manuscript.

\section{Competing interests}

The authors declare no competing or financial interests.

\section{Author contributions}

Conceptualization: P.L., J.L.B.; Methodology: P.L., F.L.-F., U.K., L.M.; Validation: P.L., F.L.-F., U.K.; Formal analysis: P.L., L.M.; Investigation: P.L., F.L.-F., U.K.; Resources: U.K., L.M.; Writing - original draft: P.L., L.M., J.L.B.; Writing - review \& editing: P.L., J.L.B.; Visualization: P.L.; Supervision: P.L., J.L.B.; Project administration: J.L.B.; Funding acquisition: P.L., L.M., J.L.B.

\section{Funding}

This work was supported by: Programa para el Desarrollo de las Ciencias Básicas (PEDECIBA) and Sistema Nacional de Investigadores- Agencia Nacional de Investigación e Innovación to P.L., U.K., L.M. and J.L.B.; FCE_1_2019_1_156365 -
Agencia Nacional de Investigación e Innovación to J.L.B.; FOCEM - Fondo para la Convergencia Estructural del Mercosur (COF 03/11). L.M. was supported by the Chan Zuckerberg Initiative.

\section{Data availability}

All the data generated in the study is presented in the manuscript.

\section{References}

Bahmad, H. F., Daouk, R., Azar, J., Sapudom, J., Teo, J. C. M., Abou-Kheir, W. and Al-Sayegh, M. (2020). Modeling adipogenesis: current and future perspective. Cells 9, 2326-2347. doi:10.3390/cells9102326

Caldovic, L., Agalliu, D. and Hackett, P. B. (1999). Position-independent expression of transgenes in zebrafish. Transgenic Res. 8, 321-334. doi:10.1023/A:1008993022331

Cao, Y. (2007). Angiogenesis modulates adipogenesis and obesity. J. Clin. Invest. 117, 2362-2368. doi:10.1172/JCl32239

Cleal, L., Aldea, T. and Chau, Y.-Y. (2017). Fifty shades of white: understanding heterogeneity in white adipose stem cells. Adipocyte 6, 205-216. doi:10.1080/ 21623945.2017 .1372871

Conover, W. J. and Iman, R. L. (1981). Rank transformations as a bridge between parametric and nonparametric statistics. Am. Stat. 35, 124-129. doi:10.2307/ 2683975

Davison, C. and Zolessi, F. R. (2021). Slit2 is necessary for optic axon organization in the zebrafish ventral midline. Cells Dev. 166, 203677. doi:10.1016/j.cdev.2021. 203677

Di Giacinto, F., De Angelis, C., De Spirito, M. and Maulucci, G. (2018). Quantitative imaging of membrane micropolarity in living cells and tissues by spectral phasors analysis. MethodsX 5, 1399-1412. doi:10.1016/j.mex.2018.10. 010

Elizondo, M. R., Arduini, B. L., Paulsen, J., MacDonald, E. L., Sabel, J. L., Henion, P. D., Cornell, R. A. and Parichy, D. M. (2005). Defective skeletogenesis with kidney stone formation in dwarf zebrafish mutant for trpm7. Curr. Biol. 15, 667-671. doi:10.1016/..cub.2005.02.050

Flynn, E. J., Trent, C. M. and Rawls, J. F. (2009). Ontogeny and nutritional control of adipogenesis in zebrafish (Danio rerio). J. Lipid Res. 50, 1641-1652. doi:10.1194/jIr.M800590-JLR200

Greenspan, P. and Fowler, S. D. (1985). Spectrofluorometric studies of the lipid probe, nile red. J. Lipid Res. 26, 781-789. doi:10.1016/S0022-2275(20)34307-8

Gupta, R. K., Arany, Z., Seale, P., Mepani, R. J., Ye, L., Conroe, H. M., Roby, Y. A., Kulaga, H., Reed, R. R. and Spiegelman, B. M. (2010). Transcriptional control of preadipocyte determination by Zfp423. Nature 464, 619-623. doi:10.1038/ nature 08816

Hammer, O., Harper, D. A. T. and Ryan, P. D. (2001). PAST: paleontological statistics software package for education and data analysis. Palaeontol. Electron 4,9 .

Harjes, U., Bridges, E., Mclntyre, A., Fielding, B. A. and Harris, A. L. (2014). Fatty acid-binding protein 4 , a point of convergence for angiogenic and metabolic signaling pathways in endothelial cells. J. Biol. Chem. 289, 23168-23176. doi:10.1074/jbc.M114.576512

Hepler, C. and Gupta, R. K. (2017). The expanding problem of adipose depot remodeling and postnatal adipocyte progenitor recruitment. Mol. Cell. Endocrinol. 445, 95-108. doi:10.1016/j.mce.2016.10.011

Hilgendorf, K. I., Johnson, C. T., Mezger, A., Rice, S. L., Norris, A. M., Demeter, J., Greenleaf, W. J., Reiter, J. F., Kopinke, D. and Jackson, P. K (2019). Omega-3 fatty acids activate ciliary FFAR4 to control adipogenesis. Cell 179, 1289-1305.e21. doi:10.1016/j.cell.2019.11.005

Hopman, A. H. N., Ramaekers, F. C. S. and Speel, E. J. M. (1998). Rapid synthesis of Biotin-, Digoxigenin-, Trinitrophenyl-, and Fluorochrome-labeled Tyramides and their application for in situ hybridization using CARD amplification J. Histochem. Cytochem. 46, 771-777. doi:10.1177/002215549804600611

Hudak, C. S., Gulyaeva, O., Wang, Y., Park, S.-M., Lee, L., Kang, C. and Sul, H. S. (2014). Pref-1 marks very early mesenchymal precursors required for adipose tissue development and expansion. Cell Rep. 8, 678-687. doi:10.1016/j.celrep. 2014.06.060

Hutchings, G., Janowicz, K., Moncrieff, L., Dompe, C., Strauss, E. Kocherova, I., Nawrocki, M. J., Kruszyna, Ł., Wasiatycz, G., Antosik, P. et al. (2020). The proliferation and differentiation of adipose-derived stem cells in neovascularization and angiogenesis. Int. J. Mol. Sci. 21, 3790-3815. doi:10.3390/ijms21113790

Imrie, D. and Sadler, K. C. (2010). White adipose tissue development in zebrafish is regulated by both developmental time and fish size. Dev. Dyn. 239, 3013-3023. doi: $10.1002 /$ dvdy. 22443

Inoue, D. and Wittbrodt, J. (2011). One for all-a highly efficient and versatile method for fluorescent immunostaining in fish embryos. PLOS ONE 6, e19713. doi:10.1371/journal.pone.0019713

Kassan, A., Herms, A., Fernández-Vidal, A., Bosch, M., Schieber, N. L., Reddy, B. J. N., Fajardo, A., Gelabert-Baldrich, M., Tebar, F., Enrich, C. et al. (2013). Acyl-CoA synthetase 3 promotes lipid droplet biogenesis in ER microdomains. J. Cell Biol. 203, 985-1001. doi:10.1083/jcb.201305142 
Kim, H. W., Shi, H., Winkler, M. A., Lee, R. and Weintraub, N. L. (2020) Perivascular adipose tissue and vascular perturbation/atherosclerosis Arterioscler. Thromb. Vasc. Biol. 40, 2569-2576. doi:10.1161/ATVBAHA.120. 312470

Kimmel, C. B., Ballard, W. W., Kimmel, S. R., Ullmann, B. and Schilling, T. F. (1995). Stages of embryonic development of the zebrafish. Dev. Dyn. 203, 253-310. doi:10.1002/aja.1002030302

Koziol, U., Rauschendorfer, T., Zanon Rodríguez, L., Krohne, G. and Brehm, K. (2014). The unique stem cell system of the immortal larva of the human parasite Echinococcus multilocularis. EvoDevo 5, 10. doi:10.1186/2041-9139-5-10

Kwan, K. M., Fujimoto, E., Grabher, C., Mangum, B. D., Hardy, M. E., Campbell, D. S., Parant, J. M., Yost, H. J., Kanki, J. P. and Chien, C.-B. (2007). The Tol2kit: a multisite gateway-based construction kit forTol2 transposon transgenesis constructs. Dev. Dyn. 236, 3088-3099. doi:10.1002/dvdy.21343

Landgraf, K., Schuster, S., Meusel, A., Garten, A., Riemer, T., Schleinitz, D. Kiess, W. and Körner, A. (2017). Short-term overfeeding of zebrafish with norma or high-fat diet as a model for the development of metabolically healthy versus unhealthy obesity. BMC Physiol. 17, 4. doi:10.1186/s12899-017-0031-x

Laprairie, R. B., Denovan-Wright, E. M. and Wright, J. M. (2017). Differential regulation of the duplicated fabp7, fabp10 and fabp11 genes of zebrafish by peroxisome proliferator activated receptors. Comp. Biochem. Physiol. B Biochem. Mol. Biol. 213, 81-90. doi:10.1016/j.cbpb.2017.08.003

Lawson, N. D. and Weinstein, B. M. (2002). In vivo imaging of embryonic vascular development using transgenic zebrafish. Dev. Biol. 248, 307-318. doi:10.1006/ dbio.2002.0711

Lemoine, A. Y., Ledoux, S. and Larger, E. (2013). Adipose tissue angiogenesis in obesity. Thromb. Haemost. 110, 661-669. doi:10.1160/TH13-01-0073

Liu, R.-Z., Saxena, V., Sharma, M. K., Thisse, C., Thisse, B., DenovanWright, E. M. and Wright, J. M. (2007). The fabp4 gene of zebrafish (Danio rerio)-genomic homology with the mammalian FABP4 and divergence from the zebrafish fabp3 in developmental expression. FEBS J. 274, 1621-1633. doi:10.1111/j.1742-4658.2007.05711.x

Loh, N. Y., Minchin, J. E. N., Pinnick, K. E., Verma, M., Todorčević, M., Denton, N., Moustafa, J. E.-S., Kemp, J. P., Gregson, C. L., Evans, D. M. et al. (2020). RSPO3 impacts body fat distribution and regulates adipose cell biology in vitro. Nat. Commun. 11, 2797. doi:10.1038/s41467-020-16592-z

Longo, M., Zatterale, F., Naderi, J., Parrillo, L., Formisano, P., Raciti, G. A Beguinot, F. and Miele, C. (2019). Adipose tissue dysfunction as determinant of obesity-associated metabolic complications. Int. J. Mol. Sci. 20, 2358-2381. doi:10.3390/ijms20092358

Lumaquin, D., Johns, E., Montal, E., Weiss, J. M., Ola, D., Abuhashem, A. and White, R. M. (2021). An in vivo reporter for tracking lipid droplet dynamics in transparent zebrafish. eLife 10, e64744. doi:10.7554/eLife.64744

Luong, Q., Huang, J. and Lee, K. Y. (2019). Deciphering white adipose tissue heterogeneity. Biology 8, 23. doi:10.3390/biology8020023

Malacrida, L. and Gratton, E. (2018). LAURDAN fluorescence and phasor plots reveal the effects of a $\mathrm{H} 2 \mathrm{O} 2$ bolus in $\mathrm{NIH}-3 \mathrm{~T} 3$ fibroblast membranes dynamics and hydration. Free Radic. Biol. Med. 128, 144-156. doi:10.1016/j.freeradbiomed. 2018.06.004

Malacrida, L., Astrada, S., Briva, A., Bollati-Fogolín, M., Gratton, E. and Bagatolli, L. A. (2016). Spectral phasor analysis of LAURDAN fluorescence in live A549 lung cells to study the hydration and time evolution of intracellular lamellar body-like structures. Biochim. Biophys. Acta (BBA) Biomembr. 1858, 2625-2635. doi:10.1016/j.bbamem.2016.07.017

Malacrida, L., Jameson, D. M. and Gratton, E. (2017). A multidimensional phasor approach reveals LAURDAN photophysics in NIH-3T3 cell membranes. Sci. Rep. 7, 9215. doi:10.1038/s41598-017-08564-Z

Mao, Y., Hong, K.-H., Liao, W., Li, L., Kim, S.-J., Xiong, Y., Nam, I.-K., Choe, S.-K and Kwak, S.-A. (2021). Generation of a novel transgenic zebrafish for studying adipocyte development and metabolic control. Int. J. Mol. Sci. 22, 3994 doi:10.3390/ijms22083994

Maulucci, G., Di Giacinto, F., De Angelis, C., Cohen, O., Daniel, B., Ferreri, C. De Spirito, M. and Sasson, S. (2018). Real time quantitative analysis of lipid storage and lipolysis pathways by confocal spectral imaging of intracellular micropolarity. Biochim. Biophys. Acta (BBA) Mol. Cell Biol. Lipids 1863, 783-793. doi:10.1016/j.bbalip.2018.04.004

Miehle, F., Möller, G., Cecil, A., Lintelmann, J., Wabitsch, M., Tokarz, J., Adamski, J. and Haid, M. (2020). Lipidomic phenotyping reveals extensive lipid remodeling during adipogenesis in human adipocytes. Metabolites 10, 217. doi:10.3390/metabo10060217
Minchin, J. E. N. and Rawls, J. F. (2017a). In vivo imaging and quantification of regional adiposity in zebrafish. In Methods in Cell Biology (ed. H.W. Detrich III, M. Westerfield and L. I. Zon). pp. 3-27. Elsevier.

Minchin, J. E. N. and Rawls, J. F. (2017b). A classification system for zebrafish adipose tissues. Dis. Models Mech. 10, 797-809. doi:10.1242/dmm.025759

Minchin, J. E. N., Dahlman, I., Harvey, C. J., Mejhert, N., Singh, M. K Epstein, J. A., Arner, P., Torres-Vázquez, J. and Rawls, J. F. (2015). Plexin D1 determines body fat distribution by regulating the type $\mathrm{V}$ collagen microenvironment in visceral adipose tissue. Proc. Natl. Acad. Sci. USA 112 4363-4368. doi:10.1073/pnas.1416412112

Olzmann, J. A. and Carvalho, P. (2019). Dynamics and functions of lipid droplets. Nat. Rev. Mol. Cell Biol. 20, 137-155. doi:10.1038/s41580-018-0085-z

Parichy, D. M., Elizondo, M. R., Mills, M. G., Gordon, T. N. and Engeszer, R. E. (2009). Normal table of postembryonic zebrafish development: staging by externally visible anatomy of the living fish. Dev. Dyn. 238, 2975-3015. doi:10.1002/dvdy.22113

Prentice, K. J., Saksi, J. and Hotamisligil, G. S. (2019). Adipokine FABP4 integrates energy stores and counterregulatory metabolic responses. J. Lipid Res. 60, 734-740. doi:10.1194/jlr.S091793

Ranjit, S., Malacrida, L., Stakic, M. and Gratton, E. (2019). Determination of the metabolic index using the fluorescence lifetime of free and bound nicotinamide adenine dinucleotide using the phasor approach. J. Biophotonics 12 e201900156. doi:10.1002/jbio.201900156

Roberts, J. A., Miguel-Escalada, I., Slovik, K. J., Walsh, K. T., Hadzhiev, Y., Sanges, R., Stupka, E., Marsh, E. K., Balciuniene, J., Balciunas, D. et al. (2014). Targeted transgene integration overcomes variability of position effects in zebrafish. Development 141, 715-724. doi:10.1242/dev.100347

Salmerón, C. (2018). Adipogenesis in fish. J. Exp. Biol. 221, jeb161588 doi:10.1242/jeb.161588

Sambrook, J., Fritsch, E. F. and Maniatis, T. (1990). Molecular Cloning: A Laboratory Manual, 2nd edn. Cold Spring Harbor Laboratory Press.

Sameni, S., Malacrida, L., Tan, Z. and Digman, M. A. (2018). Alteration in fluidity of cell plasma membrane in huntington disease revealed by spectral phasor analysis. Sci. Rep. 8, 734. doi:10.1038/s41598-018-19160-0

Schindelin, J., Arganda-Carreras, I., Frise, E., Kaynig, V., Longair, M. Pietzsch, T., Preibisch, S., Rueden, C., Saalfeld, S., Schmid, B. et al. (2012). Fiji: an open-source platform for biological-image analysis. Nat. Methods 9, 676-682. doi:10.1038/nmeth.2019

Schwalie, P. C., Dong, H., Zachara, M., Russeil, J., Alpern, D., Akchiche, N. Caprara, C., Sun, W., Schlaudraff, K.-U., Soldati, G. et al. (2018). A stromal cell population that inhibits adipogenesis in mammalian fat depots. Nature $\mathbf{5 5 9}$ 103-108. doi:10.1038/s41586-018-0226-8

Tang, Q. Q. and Lane, M. D. (2012). Adipogenesis: from stem cell to adipocyte Annu. Rev. Biochem. 81, 715-736. doi:10.1146/annurev-biochem-052110 115718

Tang, W., Zeve, D., Suh, J. M., Bosnakovski, D., Kyba, M., Hammer, R. E., Tallquist, M. D. and Graff, J. M. (2008). White fat progenitor cells reside in the adipose vasculature. Science 322, 583-586. doi:10.1126/science.1156232

Tontonoz, P., Graves, R. A., Budavari, A. I., Erdjument-Bromage, H., Lui, M., Hu, E., Tempst, P. and Spiegelman, B. M. (1994). Adipocyte-specific transcription factor ARF6 is a heterodimeric complex of two nuclear hormone receptors, PPAR7 and RXRa. Nucleic Acids Res. 22, 5628-5634. doi:10.1093/ nar/22.25.5628

Vishvanath, L. and Gupta, R. K. (2019). Contribution of adipogenesis to healthy adipose tissue expansion in obesity. J. Clin. Invest. 129, 4022-4031. doi:10.1172 JCl129191

Wang, Y., Kaiser, M. S., Larson, J. D., Nasevicius, A., Clark, K. J., Wadman, S. A., Roberg-Perez, S. E., Ekker, S. C., Hackett, P. B. McGrail, M. et al. (2010). Moesin1 and Ve-cadherin are required in endothelia cells during in vivo tubulogenesis. Development 137, 3119-3128. doi:10.1242 dev. 048785

Wang, H., Becuwe, M., Housden, B. E., Chitraju, C., Porras, A. J., Graham, M. M. Liu, X. N., Thiam, A. R., Savage, D. B., Agarwal, A. K. et al. (2016). Seipin is required for converting nascent to mature lipid droplets. eLife 5, e16582. doi:10.7554/eLife. 16582

Wilson, M. H., Ekker, S. C. and Farber, S. A. (2021). Imaging cytoplasmic lipid droplets in vivo with fluorescent perilipin 2 and perilipin 3 knockin zebrafish. eLife e66393. doi:10.7554/eLife.66393

Zwick, R. K., Guerrero-Juarez, C. F., Horsley, V. and Plikus, M. V. (2018) Anatomical, physiological, and functional diversity of adipose tissue. Cell Metab. 27, 68-83. doi:10.1016/j.cmet.2017.12.002 